\title{
TANK 41H SALTSTONE REGULATORY ANALYSES
}

February 2004

Daro Ferrara, Alex Cozzi, Christine Langton, and James Clark

Westinghouse Savannah River Company

Savannah River Site

Aiken, SC 29808

Prepared for the U.S. Department of Energy Under Contract Number DEAC09-96SR18500

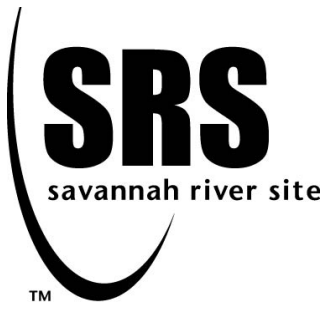


This document was prepared in conjunction with work accomplished under Contract No. DE-AC09-96SR18500 with the U. S. Department of Energy.

\section{DISCLAIMER}

This report was prepared as an account of work sponsored by an agency of the United States Government. Neither the United States Government nor any agency thereof, nor any of their employees, makes any warranty, express or implied, or assumes any legal liability or responsibility for the accuracy, completeness, or usefulness of any information, apparatus, product or process disclosed, or represents that its use would not infringe privately owned rights. Reference herein to any specific commercial product, process or service by trade name, trademark, manufacturer, or otherwise does not necessarily constitute or imply its endorsement, recommendation, or favoring by the United States Government or any agency thereof. The views and opinions of authors expressed herein do not necessarily state or reflect those of the United States Government or any agency thereof.

This report has been reproduced directly from the best available copy.

Available for sale to the public, in paper, from: U.S. Department of Commerce, National Technical Information Service, 5285 Port Royal Road, Springfield, VA 22161, phone: (800) 553-6847, fax: (703) 605-6900

email: orders@ntis.fedworld.gov

online ordering: http://www.ntis.gov/help/index.asp

Available electronically at http://www.osti.gov/bridge

Available for a processing fee to U.S. Department of Energy and its contractors, in paper, from: U.S. Department of Energy, Office of Scientific and Technical Information, P.O. Box 62, Oak Ridge, TN 37831-0062,

phone: (865)576-8401,

fax: (865)576-5728

email: $\underline{\text { reports@ adonis.osti.gov }}$ 
Key Words: Saltstone

Tank 41

Low-Level Waste

Grout

Low-Curie Salt

Retention: Permanent

\title{
TANK 41H SALTSTONE TCLP RESULTS
}

\author{
Daro Ferrara, Westinghouse Savannah River Co., 773-41A \\ Alex Cozzi, Westinghouse Savannah River Co., 773-43A \\ Christine Langton, Westinghouse Savannah River Co., 773-43A \\ James Clark, BWXT Services Inc
}

\section{FEBRUARY 2004}

Westinghouse Savannah River Company

Savannah River Site

Aiken, SC 29808

Prepared for the U.S. Department of Energy Under Contract Number DE-AC09-96SR18500

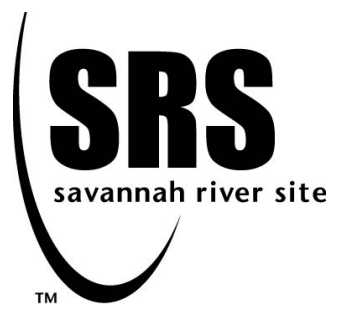




\section{ACKNOWLEDGEMENTS}

Activities described in this report were only possible because several individuals were willing to accept ownership of the task and made communication with other team members a priority. In particular, the authors would like to thank personnel at BWXS. Technical leads at BWXS were Paul Macek, Daronda Lancaster, Rick DeVault, and Ray Heinrich. In addition, at the Savannah River Site, we are thankful for the extra effort put into sample preparation and shipment by Sarah Brown, Rene Johnson, Allen Riddick, and Joe Burckhalter. Of course, the skills and customer orientation of the SRTC shield cells personnel were key to completing this task. The shielded cells personnel most directly involved were Tim Allen, Monica Miller, Debbie Burckhalter, and Martha Holmes. 


\section{TABLE OF CONTENTS}

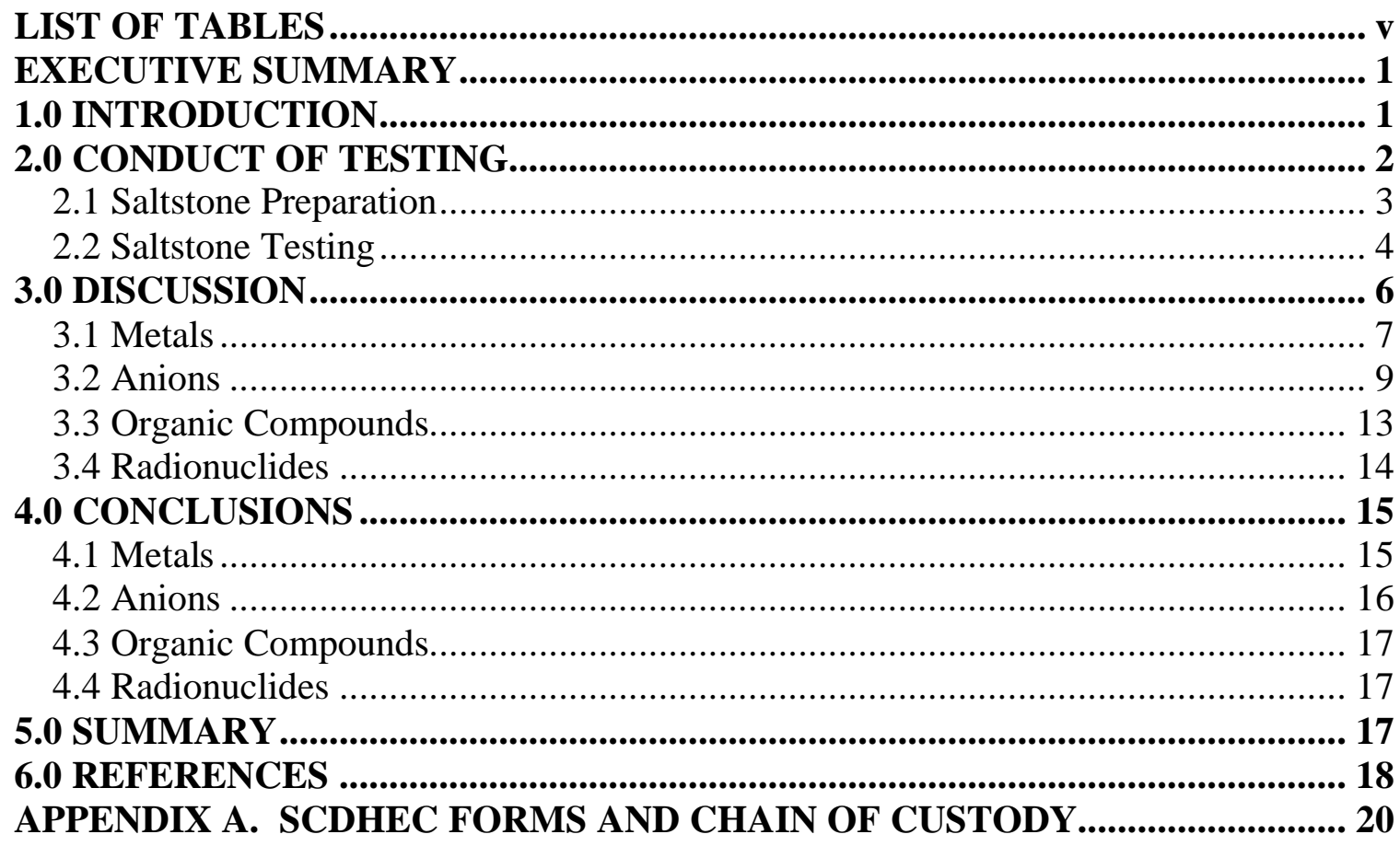

\section{LIST OF TABLES}

Table 1. Description of Tank 41H Saltstone Grout Samples.......................................... 4

Table 2. Summary of TCLP Extractions, Preparation Methods, and Analytical Methods 5

Table 3. Summary of Methods Used to Analyze Total Concentrations in the Grout........ 6

Table 4. TCLP Leachate Metal Concentrations, MDLs, and EQLs................................. 8

Table 5. TCLP Leachate Metals Preparation and Calibration Blank Results ................... 9

Table 6. TCLP Metal Matrix Spikes, Laboratory Control Samples, and Post Spikes..... 10

Table 7. Results from Analysis of Metals in the Digested Tank 41 Grout Samples....... 10

Table 8. Saltstone Metals Preparation and Calibration Blank Results ............................ 11

Table 9. Saltstone Matrix Spike, Laboratory Control Sample, and Post Spike Results .. 11

Table 10. TCLP Leachate Anion Concentrations, MDLs, and EQLs ............................ 12

Table 11. Tank 41 Saltstone Total Anion Concentrations and EQLs............................ 12

Table 12. Tank 41 Saltstone Anion Concentrations and EQLs..................................... 13

Table 13. TCLP Leachate Organic Compound Concentrations, MDLs, and EQLs ....... 14

Table 14. Organic Compound Matrix Spikes, Laboratory Control Samples, and Blanks 14

Table 15. TCLP Leachate Radionuclide Concentrations and MDAs ............................. 15 


\section{EXECUTIVE SUMMARY}

A Saltstone waste form was prepared in the Savannah River Technical Center (SRTC) shielded cells facility from a Tank $41 \mathrm{H}$ sample and Z-Area premix material. After a cure of at least 28 days, samples of the Saltstone were collected and characterized. Results showed that a Class 3 Industrial Solid Waste Landfill (ISWLF) would be required for disposal of this Tank $41 \mathrm{H}$ Saltstone waste form because of high leachate nitrate and alpha-emitting radionuclide concentrations.

\subsection{INTRODUCTION}

After evaluation of options for processing the Tank 41 salt solution, the SRS tank waste closure strategy may include immobilization of this salt solution in the SRS Saltstone facility. Tank 50 is the feed tank for the Saltstone process. In a separate study, the content of Tank 50 was sampled and immobilized in Saltstone. Results from characterization of this Tank 50 Saltstone have been documented as part of a separate report. Before initiating a treatment process for the Tank 41 salt solution, the technical basis is being examined for the disposition options.

Demonstration of a technical basis for immobilization of any waste stream in Saltstone requires

-demonstrating the waste stream solution can be processed at the SRS Saltstone facility -demonstrating the Saltstone waste form will qualify as a nonhazardous radioactive waste -demonstrating the Saltstone waste form will be compatible with the disposal facility

Processability is established when characterization of a salt solution meets the Z-Area Waste Acceptance Criteria (WAC). ${ }^{1}$ The WAC provides limits for radionuclide and chemical constituents and for physical properties. In addition, Tank $41^{2}$ and Tank $50^{3}$ Saltstone have been shown to qualify as nonhazardous radioactive waste forms. By passing the Toxicity Characterization Leaching Procedure (TCLP) test for the D-Code constituents, ${ }^{4}$ Tank 41 and 50 Saltstones have now been shown to no longer display the characteristic of metal toxicity.

Demonstrating compatibility between the waste form and the disposal facility requires that a determination be made as to whether a Class 1, 2, or 3 Industrial Solid Waste Landfill (ISWLF) is required. This is based on the leachability of constituents of potential concern (COPCs) from the waste form. Leachability is evaluated by comparison of results from TCLP analyses to SCDHEC R.61-107.16 Subpart A 16.4 Maximum Contaminant Levels (MCLs) and Primary Remediation Goals (PRGs). ${ }^{5}$ 
The objective of this report is to document results from tests performed to determine the appropriate ISWLF class for a Saltstone waste form if it were to contain Tank 41 salt solution. Results from radionuclide analyses have also been included. With the exception of radium-226, radium-228, and the total alpha emitter concentrations, these analytes are not included as COPCs in SCDHEC R61-107.16 Subpart A 16.4. Specifications for activities described in this report have been documented in the task plan for this work. ${ }^{6}$ Tank 50 Saltstone had previously been shown to require a Class 3 ISWLF. ${ }^{3}$

\subsection{CONDUCT OF TESTING}

This chapter is a summary of the approach taken to prepare and characterize the Tank 41 Saltstone samples. The Saltstone was mixed and sampled in the SRTC shielded cells facility. Samples were characterized at the BWXT Services, Inc. (BWXS) laboratory facility in Lynchburg, Virginia. BWXS is a division of BWX Technology, Inc. (BWXT). Figure 1 is a flowchart of the steps taken to prepare and characterize the Saltstone samples.

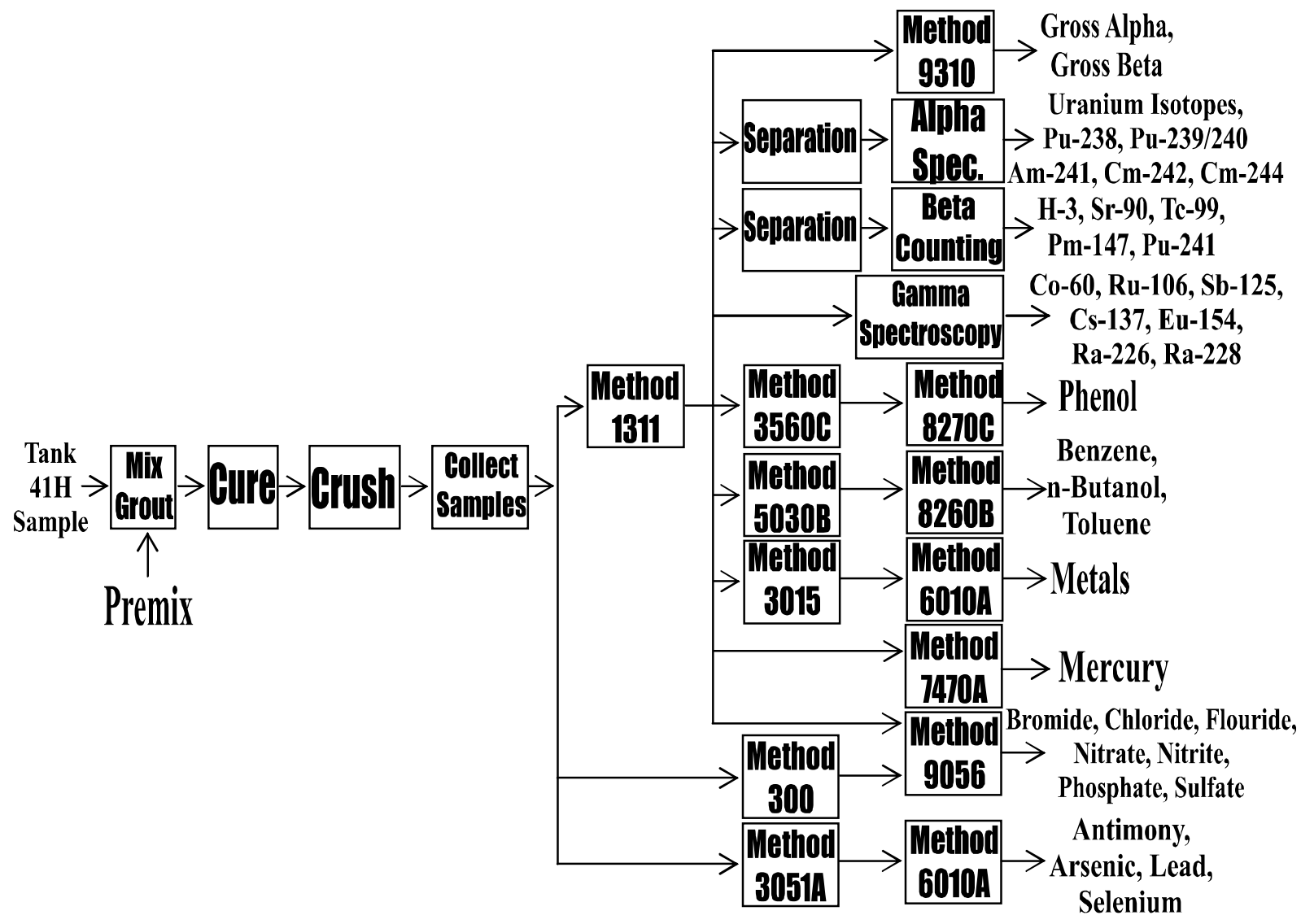

Figure 1. Flowchart of Tank 41H Saltstone Preparation and Characterization Activities 


\subsection{Saltstone Preparation}

Activities associated with preparation of the Saltstone samples were

-collection of a three-liter sample from Tank 41

-mixing of Tank 41 dissolved salt with grout-forming additives

-casting the Tank 41 Saltstone in forms for curing

-size reduction of the cured Saltstone and sample collection

-packaging and shipping of samples to BWXS

On August 25, a 3-liter sample of salt solution was collected from Tank 41H and shipped to SRTC. The sample was transferred into the SRTC shielded cells facility where Saltstone samples were prepared from the salt solution, and a premix of cement, slag and fly ash as shown in Figure 2. To accomodate other BWXS commitments and to ensure that the Tank 41 Saltstone samples did not exceed the USEPA SW-846 specified sample holding times, samples were allowed to cure for 32 days instead of 28 days. On September 29, Saltstone samples were crushed to generate material of particles with maximum diameters of less than 0.9 centimeter ( $3 / 8$ inch). To minimize radiation exposure during sample leaching and subsequent leachate preparation and analysis, BWXS requested samples be aliquoted in the SRTC shielded cells facility prior to shipment. The size-reduced material was divided into samples as described in Table 1.

\section{4 g Tank 41 Salt Solution}

Composition: $51.3 \%$ Dissolved and Undissolved Solids (207 g)

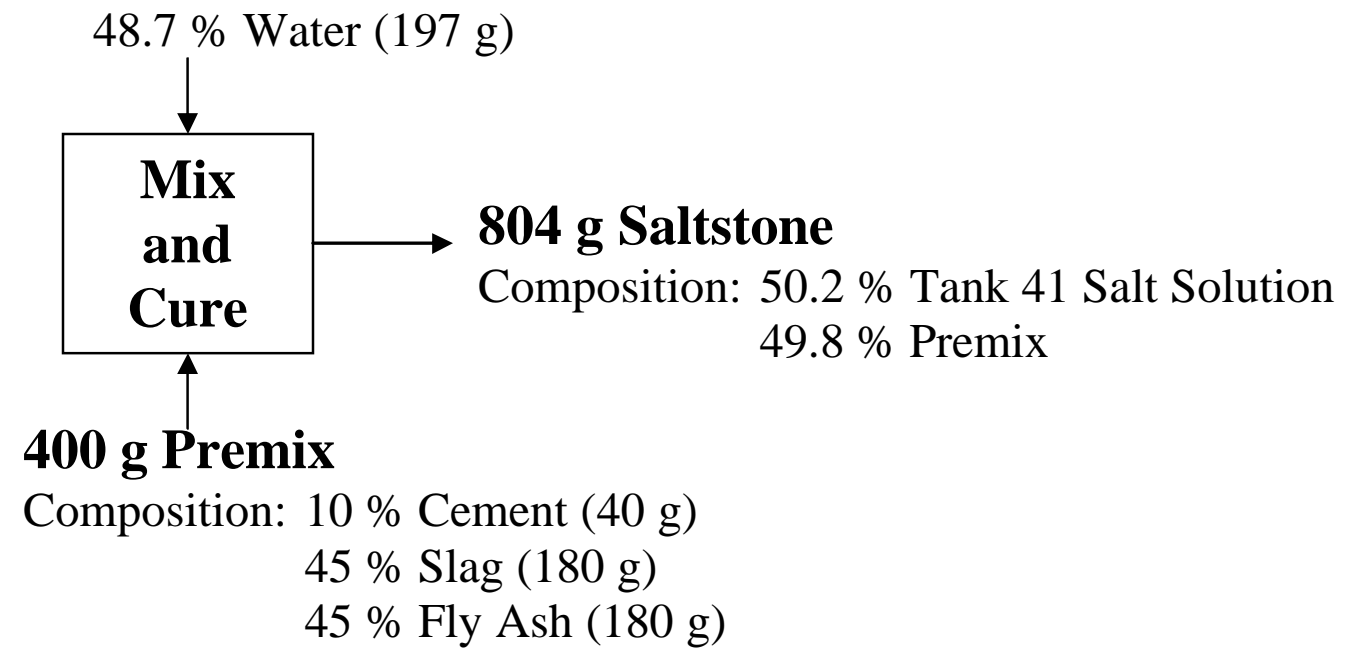

Figure 2. Components Used to Prepare the Tank 41H Saltstone Grout Samples

(Water to Premix Ratio (w/c) of 0.49) 


\subsection{Saltstone Testing}

Saltstone testing was performed by BWXS. The crushed Tank 41 Saltstone samples were first leached according to TCLP which is USEPA SW-846 Method $1311 .^{7}$ Because of interferences, analyses were not successful at demonstrating that the leachate concentrations of fluoride was beneath the SCDHEC MCL. In addition, because instrumentation was not available to perform antimony, arsenic, lead and selenium analyses by the more sensitive atomic absorption methods, analyses were not successful at demonstrating the leachate concentrations of these analytes were beneath the SCDHEC MCLs. Therefore, the total concentrations of these five analytes were determined in the Tank 41 waste form in order to demonstrate their concentrations were low enough that the MCLs could not be exceeded.

Table 1. Description of Tank 41H Saltstone Grout Samples

\begin{tabular}{|c|c|c|}
\hline SRS Sample ID & Mass (g) & Purpose of Sample \\
\hline 41-TR1 & 25.546 & TCLP Leachate Metals and Radionuclide Analyses \\
\hline 41-TR2 & 25.413 & TCLP Leachate Metals and Radionuclide Analyses \\
\hline 41-TR3 & 25.674 & TCLP Leachate Metals and Radionuclide Analyses \\
\hline 41-TM1 & 10.285 & TCLP Leachate Anion and Mercury Analyses \\
\hline 41-TM2 & 10.552 & TCLP Leachate Anion and Mercury Analyses \\
\hline 41-TM3 & 10.391 & TCLP Leachate Anion and Mercury Analyses \\
\hline 41-TM4 & 10.894 & Back-up Sample \\
\hline 41-TM5 & 11.003 & TCLP Leachate Anion Matrix Spike \\
\hline 41-TV1 & 4.157 & TCLP Leachate Volatile Organic Compound Analyses \\
\hline 41-TV2 & 4.099 & TCLP Leachate Volatile Organic Compound Analyses \\
\hline 41-TV3 & 4.371 & TCLP Leachate Volatile Organic Compound Analyses \\
\hline 41-TV4 & 4.449 & TCLP Leachate Volatile Organic Compound Matrix Spike \\
\hline 41-TV5 & 4.611 & TCLP Leachate Volatile Organic Compound Matrix Spike Duplicate \\
\hline 41-WA1 & 1.071 & Grout Total Anion Analyses \\
\hline 41-WA2 & 1.241 & Grout Total Anion Analyses \\
\hline 41-WA3 & 1.834 & Grout Total Anion Analyses \\
\hline 41-WA4 & 1.261 & Back-up Sample \\
\hline 41-WA5 & 1.101 & Back-up Sample \\
\hline 41T-E1 & 10.719 & Grout Antimony, Arsenic, Lead, and Selenium Analyses \\
\hline
\end{tabular}

\subsubsection{TCLP Analyses}

To determine compliance of the Tank 41 Saltstone waste form, TCLP (Method 1311) was performed and the leachate analyzed. TCLP leachates were treated and analyzed as follows:

-digestion and analysis of leachates for metal COPCs

-analysis of leachates for anion COPCs

-pretreatment and analysis of leachates for organic COPCs

-separation and analysis of leachate for radionuclide analytes 
On October 1, BWXS received the samples and started the TCLP extraction according to USEPA SW- $846^{7}$ Method 1311. The only exception taken to the method was use of small samples (1 to 25 grams) instead of the specified 100-gram samples. To retain the integrity of the method, the extraction was performed with "...an amount of extraction fluid equal to 20 times the weight of the solid phase."7 Semivolatile and volatile extractions were performed. The resulting leachates were filtered and $\mathrm{pH}$-adjusted according to Method 1311. Table 1 shows which samples were used in each analysis.

Aliquots from the Method 1311 leachates were prepared and analyzed according to the methods shown in Figure 1 and outlined in Table 2. In addition to the sample leachates, standard quality control samples were prepared and analyzed. These included matrix spikes, matrix spike duplicates, replicates, laboratory control samples, initial and continuing calibration standards and blanks, method blanks, digestion blanks, extraction blanks, internal standards, and mass spectrometer tune checks.

Table 2. Summary of TCLP Extractions, Preparation Methods, and Analytical Methods

\begin{tabular}{|c||c||c|c|}
\hline \multicolumn{1}{|c|}{ Analyte } & Extraction & \multicolumn{2}{c|}{ USEPA SW-846 Method } \\
\hline & & Preparation & Analysis \\
\hline Metals & Semivolatile & 3015 & $6010 \mathrm{~B}$ \\
\hline Mercury & Semivolatile & $7470 \mathrm{~A}$ & $7470 \mathrm{~A}$ \\
\hline Anions & Semivolatile & None & 9056 \\
\hline Phenol & Semivolatile & $3560 \mathrm{C}$ & $8270 \mathrm{C}$ \\
\hline Benzene, n-Butanol, Toluene & Volatile & 5030B & $8260 \mathrm{~B}$ \\
\hline Gross Alpha and Beta & Semivolatile & None & 9310 \\
\hline Alpha-Emitting Radionuclides & Semivolatile & Separations & Alpha Spectroscopy \\
\hline H-3, Sr-90, Tc-99, Pm-147, Pu-241 & Semivolatile & Separations & Beta Liquid Scintillation \\
\hline Gamma-Emitting Radionuclides & Semivolatile & Separations & Gamma Spectroscopy \\
\hline
\end{tabular}

Preparations and analyses were performed according to USEPA SW-846 methods. Although antimony, arsenic, lead, and selenium would have been analyzed by USEPA SW-846 atomic absorption methods (Methods 7041, 7060A, 7421, and 7740 for antimony, arsenic, lead, and selenium, respectively) instrumentation difficulties mandated use of results from the USEPA SW-846 ion-coupled plasma-atomic emission spectroscopy method (Method 6010B). In addition, because of difficulties with the anion matrix spike analyses, only matrix spike duplicate results have been reported. Precision has been verified by the deviation between samples rather than between the matrix spike and matrix spike duplicate.

\subsubsection{Grout Analyses}

The total antimony, arsenic, lead, selenium, and anions were determined in the grout. Table 3 is a summary of the methods used. Samples were treated and analyzed as follows:

-Digestion of grout samples for metals analyses 
-Analysis of digested grout samples for antimony, arsenic, lead, and selenium -Leaching of grout samples for anions analyses

-Analysis of grout leachate for anions

Table 3. Summary of Methods Used to Analyze Total Concentrations in the Grout

\begin{tabular}{|c|c|c|}
\hline \multicolumn{1}{|c||}{ Analyte } & \multicolumn{2}{c|}{ USEPA SW-846 Method } \\
\hline- & Preparation & Analysis \\
\hline \hline Antimony, Arsenic, Lead, and Selenium & 3051 & $6010 \mathrm{~B}$ \\
\hline Anions & ${ }^{1}$ EPA Method 300 & 9056 \\
\hline
\end{tabular}
This is a standard USEPA method but is not one of the USEPA SW-846 methods.

For the antimony, arsenic, lead, and selenium analyses, two aliquots were taken from Sample 41-TE1. These aliquots were digested using USEPA Method 3051. In addition to the nitric acid specified in Method 3051, hydrochloric acid was used in the digestion. Because of limited sample availability, triplicate aliquots were not available. The resulting duplicate digested samples were analyzed according to Method 6010B.

Aliquots from the Method 3051 digested samples were analyzed using Method 6010B. In addition to the sample leachates, standard quality control samples were prepared and analyzed. These included a matrix spike, matrix spike duplicate, laboratory control samples, post spike, initial and continuing calibration standards and blanks, a method blank and serial dilution.

In planning for these analyses, BWXS recommended additional samples be supplied for anion determinations in anticipation of acetate interference issues. Samples 41-WA1, 41WA2, 41-WA3, 41-WA4, and 41-WA5 were provided for this purpose. These samples were leached according to EPA Method 300. Although not a USEPA SW-846 method, this is a standard USEPA method for quantitatively leaching water soluble anions from a sample. Samples 41-WA4 and 41-WA5 were to be used as a matrix spike and matrix spike duplicate.

Aliquots from the EPA Method 300 leachates were analyzed using Method 9056. In addition to the sample leachates, standard quality control samples were prepared and analyzed. These included laboratory control samples, initial and continuing calibration standards and blanks, and a method blank. Due to an oversight, neither a matrix spike and nor a matrix spike duplicate was prepared.

\subsection{DISCUSSION}

Results from the analyses have been presented in this chapter. Results were summarized from the data package for these analyses. For analytes detected at concentrations too low to determine quantitatively, results have been flagged with the standard data qualifiers. For analytes that were not detected, Method Detection Limits (MDLs) or Estimated Quantitation Limits (EQLs) have been given preceded by "<". When average values were reported in this chapter, uncertainties were given as standard deviations of the triplicate results. 


\subsection{Metals}

Results have been presented in this section for analysis of metal COPCs in the TCLP leachate and of the total concentrations of antimony, arsenic, lead, and selenium in the Tank 41 Saltstone samples. Because antimony, arsenic, lead, and selenium could not be measured to concentrations below the MCLs, grout samples were digested, and the total concentrations of these four COPCs were determined. Results have been compared to the minimum concentration that would need to be present to exceed the MCLs during a TCLP analysis.

\subsubsection{Analysis of Metals in TCLP Leachate}

Results from TCLP leachate analyses have been given in Table 4. In general, results were consistent with expected values. As shown in Table 4, some analytes were detected in at least one of the blanks at concentrations similar to the sample results. USEPA Contract Laboratory Program (CLP) guidelines ${ }^{9}$ indicate that when a COPC is detected in one of the blanks and in one of the samples, the sample result can be reported as a "nondetect" if the sample result is less than five times the concentration detected in the blank. Application of the CLP 5x rule would identify these sample results as "nondetects". Blank results have been given in Subsection 3.1.2.1.

\subsubsection{Quality Assurance for Analysis of Metals in TCLP Leachate}

The following subsections include summaries of results from blanks, matrix spikes, matrix spike duplicates, laboratory control samples, and post spikes. The data package for this task also includes data for calibration verifications, low level concentration standards, interference checks, serial dilutions, instrument detection limits, interelement correction factors, linear range analysis and concentrations that were used for determining the recoveries.

\subsubsection{Blanks}

Blank concentrations have been given in Table 5. Several analytes were detected at concentrations too low to be considered quantitative. The high sodium concentration in the extraction blank was expected because results of the TCLP extraction fluid determination section identified the appropriate extraction fluid to be Extraction Fluid 1. Extraction Fluid 1 is a $\mathrm{pH} 4.93$ buffer made from acetic acid and sodium hydroxide. Barium and zinc were detected above the EQL in the extraction blank but at trace levels. Beryllium was also detected above the EQL but at trace levels in some calibration blanks. Results were flagged if blank concentrations were high enough to apply the $\operatorname{CLP}^{9} 5 \mathrm{x}$ rule.

\subsubsection{Matrix Spikes, Laboratory Control Samples, and Post Spikes}

Results from the matrix spike, matrix spike duplicate, laboratory control samples, and post spike have been given in Table 6. All matrix spike and matrix spike duplicate recoveries met specifications except for aluminum and iron. All relative percent differences between matrix spikes and matrix spike duplicates met specifications. All post spike results were acceptable 
except lithium. Although two laboratory control samples have been given in Table 6, PB MS 640-45 was the applicable laboratory control sample for all analytes except mercury. For mercury, LCS P093-500 640-88 was the applicable laboratory control sample. The recovery for this laboratory control sample has also been given in Table 6 .

Table 4. TCLP Leachate Metal Concentrations, MDLs, and EQLs

\begin{tabular}{|c|c|c|c|c|c|c|c|c|}
\hline- & \multicolumn{4}{|c|}{ Limits (mg/L) } & \multicolumn{4}{|c|}{ Sample Results $(\mathrm{mg} / \mathrm{L})$} \\
\hline- & Regu & tory & Analyti & 1 Limits & Sample 1 & Sample 2 & Sample 3 & Average \\
\hline- & MCL & PRG & ${ }^{\mathrm{T}} \mathrm{MDL}$ & ${ }^{1} \mathrm{EQL}$ & - & - & - & - \\
\hline Date & 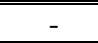 & 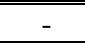 & $2-$ & $2-$ & 9/29/03 & 9/29/03 & 9/29/03 & - \\
\hline SRS ID & - & - & - & - & 41-TR1 & $41-\mathrm{TR} 2$ & 41-TR3 & - \\
\hline BWXS ID & - & - & - & - & 0310002-17 & $0310002-18$ & $0310002-19$ & - \\
\hline As & 0.01 & - & 0.012 & 0.12 & $\begin{array}{l}<0.012 \\
\end{array}$ & 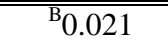 & $\begin{array}{l}{ }^{\mathrm{B}} 0.016 \\
\end{array}$ & $\overline{\overline{\mathrm{B}} 0.018 \pm 0.0050}$ \\
\hline $\mathbf{B a}$ & 2 & - & $1.2 \times 10^{-3}$ & 0.012 & ${ }^{\mathrm{Y}} 0.122$ & $\mathrm{Y}_{0.110}$ & $\mathrm{Y}_{0.152}$ & ${ }^{\mathrm{Y}_{0}} 0.128 \pm 0.022$ \\
\hline $\mathbf{C d}$ & $5 \times 10^{-3}$ & - & $1.6 \times 10^{-3}$ & 0.016 & $<1.6 \times 10^{-3}$ & ${ }^{\mathrm{YB}} 2.78 \times 10^{-3}$ & ${ }^{\mathrm{YB}} 2.11 \times 10^{-3}$ & ${ }^{\mathrm{YB}} 2.4 \pm 0.67 \times 10^{-3}$ \\
\hline $\mathbf{C r}$ & 0.1 & - & $3.2 \times 10^{-3}$ & 0.032 & ${ }^{\mathrm{B}} 0.0268$ & ${ }^{\mathrm{B}} 0.0276$ & ${ }^{\mathrm{B}} 0.0274$ & ${ }^{\mathrm{B}} 0.0272 \pm 0.00042$ \\
\hline $\mathbf{P b}$ & 0.015 & - & 0.028 & 0.28 & $<0.028$ & $<0.028$ & $<0.028$ & $<0.028$ \\
\hline $\mathbf{H g}$ & $2 \times 10^{-3}$ & - & $1.4 \times 10^{-4}$ & $1.4 \times 10^{-3}$ & $<1.4 \times 10^{-4}$ & ${ }^{\mathrm{YB}} 1.8 \times 10^{-4}$ & ${ }^{\mathrm{YB}} 8.1 \times 10^{-4}$ & ${ }^{\mathrm{YB}} 5 \pm 6 \times 10^{-4}$ \\
\hline $\mathbf{S e}$ & 0.05 & - & 0.028 & 0.28 & ${ }^{\mathrm{B}} 0.156$ & ${ }^{\mathrm{B}} 0.150$ & ${ }^{\mathrm{B}} 0.163$ & ${ }^{\mathrm{B}} 0.156 \pm 0.0065$ \\
\hline $\mathbf{A g}$ & 0.1 & - & $5.4 \times 10^{-3}$ & $5.4 \times 10^{-2}$ & $<5.4 \times 10^{-3}$ & $<5.4 \times 10^{-3}$ & $<5.4 \times 10^{-3}$ & $<5.4 \times 10^{-3}$ \\
\hline $\mathbf{A l}$ & - & 36 & 0.030 & 0.30 & 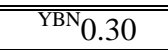 & 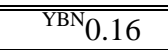 & 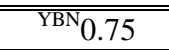 & $\overline{\mathrm{YBN}} 0.40 \pm 0.31$ \\
\hline $\mathbf{S b}$ & $6 \times 10^{-3}$ & - & 0.028 & 0.28 & $<0.028$ & $<0.028$ & $<0.028$ & $<0.028$ \\
\hline $\mathbf{B e}$ & $4 \times 10^{-3}$ & - & $1.7 \times 10^{-4}$ & $1.7 \times 10^{-3}$ & $<1.7 \times 10^{-4}$ & $1.1 \times 10^{-3}$ & $<1.7 \times 10^{-4}$ & $1.1 \times 10^{-3}$ \\
\hline $\mathbf{B}$ & - & 3.3 & 0.018 & 0.18 & 0.517 & 0.483 & 0.370 & $0.46 \pm 0.077$ \\
\hline Co & - & 2.2 & $2.6 \times 10^{-3}$ & 0.026 & $<2.6 \times 10^{-3}$ & $<2.6 \times 10^{-3}$ & $<2.6 \times 10^{-3}$ & $<2.6 \times 10^{-3}$ \\
\hline $\mathbf{C u}$ & 1.3 & - & $5.9 \times 10^{-3}$ & 0.059 & $<5.9 \times 10^{-3}$ & $<5.9 \times 10^{-3}$ & $<5.9 \times 10^{-3}$ & $<5.9 \times 10^{-3}$ \\
\hline $\mathbf{F e}$ & 0.3 & - & $5.1 \times 10^{-3}$ & 0.051 & $\mathrm{YBN} 0.044$ & ${ }^{\mathrm{YN}} 0.071$ & ${ }^{\mathrm{YN}} 0.098$ & $\mathrm{YBN}_{0.071 \pm 0.027}$ \\
\hline $\mathbf{L i}$ & - & 0.73 & 0.011 & 0.11 & 0.763 & 0.766 & 0.710 & $0.75 \pm 0.032$ \\
\hline Mn & 0.05 & - & $1.2 \times 10^{-3}$ & 0.012 & $\mathrm{Y}_{3.8 \times 10^{-3}}$ & $\mathrm{Y}_{2.9 \times 10^{-3}}$ & $\begin{array}{l}\mathrm{Y}_{0} 0.0127 \\
\end{array}$ & ${ }^{\mathrm{YB}} 6.5 \pm 5.4 \times 10^{-3}$ \\
\hline Mo & - & 0.18 & 0.023 & 0.23 & 0.486 & 0.484 & 0.442 & $0.47 \pm 0.024$ \\
\hline $\mathbf{N i}$ & - & 0.73 & $6.9 \times 10^{-3}$ & 0.069 & $<6.9 \times 10^{-3}$ & $<6.9 \times 10^{-3}$ & $<6.9 \times 10^{-3}$ & $<6.9 \times 10^{-3}$ \\
\hline $\mathbf{K}$ & - & - & 0.13 & 1.3 & 61.3 & 60.7 & 57.7 & $60 \pm 1.9$ \\
\hline $\mathbf{N a}$ & - & - & 0.028 & 0.28 & $\mathrm{Y}_{3.96 \times 10^{3}}$ & $\mathrm{Y}_{3.94 \times 10^{3}}$ & $\mathrm{Y}_{3.66 \times 10^{3}}$ & $\mathrm{Y}_{3.9 \pm 0.17 \times 10^{3}}$ \\
\hline $\mathbf{S i}$ & - & - & 0.029 & 0.29 & 35.9 & 37.6 & 38.1 & $37 \pm 1.2$ \\
\hline $\mathbf{S r}$ & - & 22 & $1.3 \times 10^{-3}$ & 0.013 & 0.957 & 0.912 & 1.02 & $0.96 \pm 0.054$ \\
\hline $\mathbf{Z n}$ & 5 & - & $4.0 \times 10^{-3}$ & 0.040 & $<4.0 \times 10^{-3}$ & $\mathrm{Y}_{0.052}$ & $<4.0 \times 10^{-3}$ & ${ }^{\mathrm{Y}} 0.052$ \\
\hline
\end{tabular}

- Indicates a location in the table for which an entry would not be appropriate.

${ }^{1}$ MDLs were from USEPA SW-846 MDL studies with standards in water. EQLs were estimated as ten times the MDLs.

${ }^{\mathrm{B}}$ Analyte is present at a concentration above the MDL but less than the EQL.

${ }^{N}$ The matrix spike and matrix spike duplicate recoveries for these analytes were not within specified QA limits.

Y Application of the CLP 5x rule would make this result a "nondetect" due to similar concentrations in the extraction blank.

\subsubsection{Analysis of Antimony, Arsenic, Lead, and Selenium in Tank 41 Saltstone Samples}

Results from analysis of the digested Tank 41 grout have been given in Table 7 for the analysis of antimony, arsenic, lead, and selenium. The MCLs given in Table 7 are the concentrations in the grout that would produce a TCLP leachate equal to the SCDHEC MCLs if all of the COPC were to be leached during a TCLP. Lead and selenium results indicated that these COPCs were present at concentrations greater than the MCLs. Because the selenium results were so close to the detection limits and because selenium was not detected in the Tank 41 salt solution, ${ }^{10}$ the selenium results are suspect and may have been caused 
from interference by another analyte. Antimony and arsenic were determined to be below the MDLs; however, the MDLs were not low enough to establish whether the analytes were present in concentrations above the MCLs. Results from the blank analyses have been given in Table 8. In addition, recoveries and RPDs have been given in Table 9.

Table 5. TCLP Leachate Metals Preparation and Calibration Blank Results

\begin{tabular}{|c|c|c|c|c|c|c|c|c|}
\hline \multirow{3}{*}{$\begin{array}{c}\text { Analyte } \\
-\end{array}$} & \multicolumn{2}{|c|}{ Preparation Blanks (mg/L) } & \multicolumn{6}{|c|}{ Calibration Blanks (mg/L) } \\
\hline & Digestion & Extraction & Initial & & & Continuing & & \\
\hline & - & - & - & 1 & 2 & 3 & 4 & 5 \\
\hline As & $<<0.011$ & $<00.011$ & $<<0.011$ & $<<0.011$ & $<00.011$ & $<0.011$ & $<0.011$ & $<<0.011$ \\
\hline $\mathbf{B a}$ & $<1.1 \times 10^{-3}$ & ${ }^{\mathrm{Y}} 0.0246$ & $<1.1 \times 10^{-3}$ & $<1.1 \times 10^{-3}$ & $<1.1 \times 10^{-3}$ & $<1.1 \times 10^{-3}$ & $<1.1 \times 10^{-3}$ & $<1.1 \times 10^{-3}$ \\
\hline Cd & ${ }^{\mathrm{B}} 2.2 \times 10^{-3}$ & ${ }^{\mathrm{YB}} 1.6 \times 10^{-3}$ & ${ }^{\mathrm{B}} 1.8 \times 10^{-3}$ & ${ }^{\mathrm{B}} 2.5 \times 10^{-3}$ & ${ }^{\mathrm{B}} 3.4 \times 10^{-3}$ & ${ }^{\mathrm{B}} 1.4 \times 10^{-3}$ & ${ }^{\mathrm{B}} 5.0 \times 10^{-3}$ & ${ }^{\mathrm{B}} 2.8 \times 10^{-3}$ \\
\hline $\mathrm{Cr}$ & $<2.9 \times 10^{-3}$ & ${ }^{\mathrm{B}} 4.0 \times 10^{-3}$ & ${ }^{\mathrm{B}} 3.5 \times 10^{-3}$ & ${ }^{\mathrm{B}} 4.7 \times 10^{-3}$ & $<2.9 \times 10^{-3}$ & $<2.9 \times 10^{-3}$ & ${ }^{\mathrm{B}} 6.0 \times 10^{-3}$ & ${ }^{\mathrm{B}} 5.0 \times 10^{-3}$ \\
\hline $\mathbf{P b}$ & $<0.025$ & $<0.025$ & $<0.025$ & $<0.025$ & $<0.025$ & $<0.025$ & $<0.025$ & $<0.025$ \\
\hline $\mathrm{Hg}$ & $<7 \times 10^{-5}$ & ${ }^{\mathrm{YB}} 1.2 \times 10^{-4}$ & $<7 \times 10^{-5}$ & $<7 \times 10^{-5}$ & $<7 \times 10^{-5}$ & - & - & - \\
\hline Se & $<0.025$ & $<0.025$ & $<0.025$ & $<0.025$ & $<0.025$ & $<0.025$ & $<0.025$ & $<0.025$ \\
\hline Ag & $<4.9 \times 10^{-3}$ & ${ }^{\mathrm{B}} 5.2 \times 10^{-3}$ & ${ }^{\mathrm{B}} 5.0 \times 10^{-3}$ & $<4.9 \times 10^{-3}$ & $<4.9 \times 10^{-3}$ & $<4.9 \times 10^{-3}$ & $<4.9 \times 10^{-3}$ & $<4.9 \times 10^{-3}$ \\
\hline Al & ${ }^{\mathrm{B}} 0.049$ & 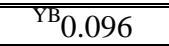 & $<<0.027$ & $<<0.027$ & $<<<0.027$ & $<<0.027$ & $<<0.027$ & $<<0.027$ \\
\hline Sb & $<0.025$ & $<0.025$ & $<0.025$ & $<0.025$ & $<0.025$ & $<0.025$ & $<0.025$ & $<0.025$ \\
\hline $\mathbf{B e}$ & $<1.5 \times 10^{-4}$ & ${ }^{\mathrm{B}} 2.0 \times 10^{-4}$ & ${ }^{\mathrm{B}} 9.0 \times 10^{-4}$ & $1.6 \times 10^{-3}$ & $2.0 \times 10^{-3}$ & ${ }^{\mathrm{B}} 8.0 \times 10^{-4}$ & $3.3 \times 10^{-3}$ & ${ }^{\mathrm{B}} 1.4 \times 10^{-3}$ \\
\hline B & $<0.016$ & $<0.15$ & $<0.016$ & $<0.016$ & $<0.016$ & $<0.016$ & - & - \\
\hline Co & $<2.3 \times 10^{-3}$ & $<2.3 \times 10^{-3}$ & $<2.3 \times 10^{-3}$ & $<2.3 \times 10^{-3}$ & $<2.3 \times 10^{-3}$ & $<2.3 \times 10^{-3}$ & $<2.3 \times 10^{-3}$ & $<2.3 \times 10^{-3}$ \\
\hline $\mathbf{C u}$ & $<5.3 \times 10^{-3}$ & 0.020 & $<5.3 \times 10^{-3}$ & $<5.3 \times 10^{-3}$ & $<5.3 \times 10^{-3}$ & $<5.3 \times 10^{-3}$ & - & - \\
\hline $\mathbf{F e}$ & $<4.6 \times 10^{-3}$ & ${ }^{\mathrm{YB}} 0.045$ & $<4.6 \times 10^{-3}$ & $<4.6 \times 10^{-3}$ & $<4.6 \times 10^{-3}$ & $<4.6 \times 10^{-3}$ & $<4.6 \times 10^{-3}$ & $<4.6 \times 10^{-3}$ \\
\hline $\mathbf{L i}$ & $<9.7 \times 10^{-3}$ & $<9.7 \times 10^{-3}$ & $<9.7 \times 10^{-3}$ & $<9.7 \times 10^{-3}$ & $<9.7 \times 10^{-3}$ & $<9.7 \times 10^{-3}$ & - & - \\
\hline Mn & $<1.1 \times 10^{-3}$ & ${ }^{\mathrm{YB}} 6.0 \times 10^{-3}$ & ${ }^{\mathrm{B}} 1.6 \times 10^{-3}$ & ${ }^{\mathrm{B}} 1.4 \times 10^{-3}$ & ${ }^{\mathrm{B}} 1.6 \times 10^{-3}$ & $<1.1 \times 10^{-3}$ & ${ }^{B} 3.2 \times 10^{-3}$ & ${ }^{\mathrm{B}} 1.6 \times 10^{-3}$ \\
\hline Mo & $<0.021$ & $<0.021$ & ${ }^{\mathrm{B}} 0.027$ & $<0.021$ & $<0.021$ & $<0.021$ & - & - \\
\hline $\mathbf{N i}$ & $<6.2 \times 10^{-3}$ & $<6.2 \times 10^{-3}$ & $<6.2 \times 10^{-3}$ & $<6.2 \times 10^{-3}$ & $<6.2 \times 10^{-3}$ & $<6.2 \times 10^{-3}$ & $<6.2 \times 10^{-3}$ & $<6.2 \times 10^{-3}$ \\
\hline $\mathbf{K}$ & $<0.12$ & ${ }^{\mathrm{B}} 0.35$ & $<0.12$ & $<0.12$ & $<0.12$ & $<0.12$ & $<0.12$ & $<0.12$ \\
\hline $\mathbf{N a}$ & ${ }^{\mathrm{B}} 0.035$ & ${ }^{\mathrm{Z}} 1.318 \times 10^{3}$ & ${ }^{\mathrm{B}} 0.036$ & ${ }^{\mathrm{B}} 0.026$ & ${ }^{\mathrm{B}} 0.065$ & ${ }^{\mathrm{B}} 0.042$ & ${ }^{\mathrm{B}} 0.054$ & ${ }^{\mathrm{B}} 0.032$ \\
\hline $\mathbf{S i}$ & 0.457 & 0.451 & $<0.026$ & $<0.026$ & $<0.026$ & $<0.026$ & - & - \\
\hline $\mathrm{Sr}$ & $<1.2 \times 10^{-3}$ & 0.0136 & ${ }^{\mathrm{B}} 1.6 \times 10^{-3}$ & $<1.2 \times 10^{-3}$ & ${ }^{\mathrm{B}} 1.7 \times 10^{-3}$ & ${ }^{\mathrm{B}} 1.9 \times 10^{-3}$ & - & - \\
\hline $\mathbf{Z n}$ & $<3.6 \times 10^{-3}$ & ${ }^{\mathrm{Y}} 0.0381$ & $<3.6 \times 10^{-3}$ & $<3.6 \times 10^{-3}$ & $<3.6 \times 10^{-3}$ & $<3.6 \times 10^{-3}$ & $<3.6 \times 10^{-3}$ & $<3.6 \times 10^{-3}$ \\
\hline
\end{tabular}

- Indicates a location in the table for which an entry would not be appropriate.

${ }^{B}$ Analyte was present at a concentration above the MDL but less than the EQL.

Y Application of the CLP 5x rule to this value would indicate that the sample result should be considered a "nondetect".

$\mathrm{Z}$ The high the extraction blank sodium concentration was due to one of the TCLP leachate components (sodium hydroxide).

\subsection{Anions}

Toxicity Characteristic Leaching Procedure (TCLP) leachate anion concentrations from the Tank 41 Saltstone waste form have been presented in this section. Because acetate in the TCLP leachate interfered with the fluoride determination, BWXS also determined the anion concentrations in the grout waste form itself by leaching the anions from a sample using EPA Method 300 and determining the anion concentrations in this leachate. 
Table 6. TCLP Metal Matrix Spikes, Laboratory Control Samples, and Post Spikes

\begin{tabular}{|c|c|c|c|c|c|c|c|c|}
\hline \multirow{3}{*}{$\begin{array}{c}\text { Analyte } \\
-\end{array}$} & \multicolumn{5}{|c|}{$\begin{array}{c}\text { Matrix Spikes } \\
\end{array}$} & \multicolumn{3}{|c|}{ Recovery $(\%)$} \\
\hline & \multicolumn{2}{|c|}{ Concentrations (mg/L) } & \multicolumn{2}{|c|}{${ }^{1}$ Recovery (\%) } & \multirow{2}{*}{$\begin{array}{c}{ }^{2} \text { RPD (\%) } \\
-\end{array}$} & \multicolumn{2}{|c|}{ Laboratory Control Samples } & \multirow[t]{2}{*}{ PS } \\
\hline & Sample & Spike Added & MS & MSD & & LCS P093-500 640-88 & PB MS 640-45 & \\
\hline As & ${ }^{\mathrm{B}} 0.014$ & 1.000 & 86.8 & 86.5 & 0.3 & 100.4 & 99.2 & 102.4 \\
\hline $\mathbf{B a}$ & 0.137 & 1.000 & 76.2 & 75.5 & 0.8 & 101.1 & 97.6 & 89.3 \\
\hline Cd & ${ }^{\mathrm{B}} 1.9 \times 10^{-3}$ & 1.000 & 79.9 & 79.3 & 0.8 & 96.6 & 99.5 & 92.9 \\
\hline $\mathbf{C r}$ & ${ }^{\mathrm{B}} 0.025$ & 1.000 & 78.6 & 78.1 & 0.6 & 111 & 99.7 & 92.1 \\
\hline $\mathbf{P b}$ & $<0.025$ & 1.000 & 76.8 & 78.5 & 2.2 & 94.7 & 100.0 & 92.9 \\
\hline $\mathrm{Hg}$ & ${ }^{\mathrm{B}} 4.1 \times 10^{-4}$ & $5.0 \times 10^{-3}$ & 106.9 & 105.9 & 0.9 & 107.6 & - & - \\
\hline Se & ${ }^{\mathrm{B}} 0.15$ & 1.000 & 90.4 & 88.5 & 1.8 & 97.7 & 98.5 & 108 \\
\hline Ag & $<4 . .9 \times 10^{-3}$ & 1.000 & 80.9 & 80.5 & 0.5 & 100.7 & 98.3 & 90.6 \\
\hline Al & 0.672 & 1.000 & $\mathrm{~N}_{31.7}$ & $\mathrm{~N}_{30.1}$ & 1.7 & 103.5 & 96.9 & 91.2 \\
\hline $\mathbf{S b}$ & $<0.025$ & 1.000 & 86.3 & 86.5 & 0.2 & 90.9 & 99.1 & 101.7 \\
\hline $\mathbf{B e}$ & $<1.5 \times 10^{-4}$ & 1.000 & 83.1 & 82.2 & 1.1 & 101.1 & 100.0 & 96.5 \\
\hline B & 0.333 & 1.000 & 92.9 & 89.0 & 3.1 & 115 & 103.0 & 101.1 \\
\hline Co & $<2.3 \times 10^{-3}$ & 1.000 & 79.2 & 79.1 & 0.2 & 104.1 & 101.0 & 92.4 \\
\hline $\mathbf{C u}$ & $<5.3 \times 10^{-3}$ & 1.000 & 83.7 & 82.7 & 1.1 & 133 & 99.5 & 97.3 \\
\hline $\mathbf{F e}$ & 0.0885 & 1.000 & ${ }^{\mathrm{N}} 69.0$ & ${ }^{\mathrm{N}} 67.7$ & 1.7 & 106.8 & 105.0 & 92.1 \\
\hline $\mathbf{L i}$ & 0.639 & 1.000 & 113.9 & 114.7 & 0.4 & - & 105.0 & ${ }^{\mathrm{N}} 134$ \\
\hline Mn & 0.0114 & 1.000 & 76.4 & 75.9 & 0.6 & 97.5 & 97.3 & 90.2 \\
\hline Mo & 0.398 & 1.000 & 96.7 & 93.6 & 2.3 & 113 & 103.0 & 104.6 \\
\hline $\mathrm{Ni}$ & $<6.2 \times 10^{-3}$ & 1.000 & 79.4 & 79.7 & 0.3 & 103 & 102.0 & 92.9 \\
\hline $\mathbf{K}$ & 51.90 & 10.00 & ${ }^{\mathrm{Y}} 60.4$ & ${ }^{Y} 54.5$ & 1.0 & - & 99.3 & 72.0 \\
\hline $\mathbf{N a}$ & - & - & - & - & - & - & 103.0 & - \\
\hline $\mathbf{S i}$ & 33.69 & 1.000 & - & - & 1.8 & - & 102.8 & - \\
\hline $\mathrm{Sr}$ & 0.915 & 1.000 & 97.9 & 97.0 & 0.5 & 97.2 & 97.6 & 109.4 \\
\hline $\mathbf{Z n}$ & $<3.6 \times 10^{-3}$ & 1.000 & 85.9 & 85.1 & 0.9 & 98.0 & 99.9 & 90.6 \\
\hline
\end{tabular}

- Indicates a location in the table for which an entry would not be appropriate.

${ }^{1}$ The acceptable range for matrix spike and matrix spike duplicate recoveries was from 75 to 125 percent.

${ }^{2}$ To be acceptable, RPDs had to be less than $20 \%$.

${ }^{\mathrm{B}}$ Analyte is present at a concentration above the MDL but less than the EQL.

${ }^{N}$ This recovery was outside of established acceptance limits. Sample results have been flagged in previous tables.

${ }^{Y}$ Low value due to low spike relative to sample concentration. Since post spike was acceptable, no qualifier was warranted.

Table 7. Results from Analysis of Metals in the Digested Tank 41 Grout Samples

\begin{tabular}{|c|c|c|c|c|c|c|c|c|}
\hline- & MCL (mg/kg) & \multicolumn{3}{|c|}{ Sample $1(\mathrm{mg} / \mathrm{kg})$} & \multicolumn{3}{|c|}{ Sample $2(\mathrm{mg} / \mathrm{kg})$} & Average (mg/kg) \\
\hline Date & - & \multicolumn{3}{|c|}{$9 / 29 / 03$} & \multicolumn{3}{|c|}{$9 / 29 / 03$} & - \\
\hline SRS ID & - & \multicolumn{3}{|c|}{ 41-TE1 } & \multicolumn{3}{|c|}{ 41-TE2 } & - \\
\hline BWXS ID & - & \multicolumn{3}{|c|}{ 0310002-01A } & \multicolumn{3}{|c|}{ 0310002-01A DUP } & - \\
\hline & & ${ }^{1} \mathrm{MDL}$ & $\begin{array}{l}{ }^{1} \mathrm{EQL} \\
\end{array}$ & Result & ${ }^{\mathrm{T}} \mathrm{MDL}$ & 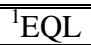 & Result & - \\
\hline As & 0.2 & 2.3 & 23 & ${ }^{\mathrm{YB}} 11.3$ & 2.6 & 26 & ${ }^{\mathrm{YB}} 10.5$ & ${ }^{\mathrm{YB}} 10.9 \pm 0.80$ \\
\hline $\mathbf{P b}$ & 0.3 & 6.4 & 64 & ${ }^{\mathrm{B}} 15.5$ & 7.2 & 72 & ${ }^{\mathrm{B}} 19.1$ & ${ }^{\mathrm{B}} 17 \pm 3.6$ \\
\hline Se & 0.1 & 5.3 & 53 & ${ }^{\mathrm{B}} 7.0$ & 6.0 & 60 & ${ }^{\mathrm{B}} 9.9$ & ${ }^{\mathrm{B}} 8.4 \pm 2.9$ \\
\hline 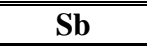 & 0.012 & 2.7 & 27 & $2<2.7$ & 3.0 & 30 & $2<3.0$ & $<<2.7$ \\
\hline
\end{tabular}

- Indicates a location in the table for which an entry would not be appropriate.

${ }^{1}$ MDLs were from USEPA SW-846 MDL studies with standards in water. EQLs were estimated as ten times the MDLs.

${ }^{B}$ Analyte is present at a concentration above the MDL but less than the EQL.

Y Application of the CLP 5x rule would make this result a "nondetect" due to similar concentrations in the extraction blank. 
Table 8. Saltstone Metals Preparation and Calibration Blank Results

\begin{tabular}{|c||c||c||c|c|c|c|}
\hline \multicolumn{1}{|c||}{ Analyte } & Digestion Blank (mg/L) & \multicolumn{5}{|c|}{ Calibration Blanks (mg/L) } \\
\hline- & - & Initial & \multicolumn{4}{|c|}{ Continuing } \\
\hline- & - & - & 1 & 2 & 3 & 4 \\
\hline As & $<0.014$ & $<0.014$ & ${ }^{\mathrm{YB}} 0.015$ & ${ }^{\mathrm{YB}} 0.016$ & $<0.014$ & $<0.014$ \\
\hline Pb & $<0.037$ & $<0.037$ & $<0.037$ & $<0.037$ & $<0.037$ & $<0.037$ \\
\hline Se & $<0.031$ & $<0.031$ & $<0.031$ & $<0.031$ & $<0.031$ & $<0.031$ \\
\hline Sb & $<0.016$ & $<0.016$ & $<0.016$ & $<0.016$ & $<0.016$ & $<0.016$ \\
\hline
\end{tabular}

- Indicates a location in the table for which an entry would not be appropriate.

${ }^{B}$ Analyte was present at a concentration above the MDL but less than the EQL.

Y Application of the CLP 5x rule to this value would indicate that the sample result should be considered a "nondetect".

Table 9. Saltstone Matrix Spike, Laboratory Control Sample, and Post Spike Results

\begin{tabular}{|c||c|c||c|c||c||c|c||c|}
\hline \multicolumn{1}{|c||}{ Analyte } & \multicolumn{4}{c||}{ Matrix Spikes } & \multicolumn{3}{c|}{ Recovery (\%) } \\
\hline \multicolumn{1}{|||}{} & Concentrations (mg/L) & ${ }^{1}$ Recovery (\%) & ${ }^{\mathbf{2}}$ RPD (\%) & \multicolumn{2}{|c||}{ Laboratory Control Samples } & ${ }^{1}$ PS \\
\hline- & Sample & Spike Added & MS & MSD & - & Result & Control Limits & - \\
\hline \hline As & ${ }^{\mathrm{B}} 0.0652$ & 4.000 & 89.0 & 77.7 & 0.3 & 96.9 & 75 to 125 & 102.3 \\
\hline Pb & ${ }^{\mathrm{B}} 0.0891$ & 1.000 & 84.6 & 90.0 & 2.2 & 98.5 & 81 to 119 & 94.4 \\
\hline Se & ${ }^{\mathrm{B}} 0.040$ & 4.000 & 88.2 & 85.5 & 1.8 & 111.0 & 67 to 133 & 99.6 \\
\hline \hline Sb & $<0.016$ & 1.000 & 80.5 & 89.8 & 0.2 & 67.8 & 50 to 150 & 100.5 \\
\hline
\end{tabular}

- Indicates a location in the table for which an entry would not be appropriate

${ }^{1}$ The acceptable range for matrix spike, matrix spike duplicate, and post spike recoveries was from 75 to 125 percent.

${ }^{2}$ To be acceptable, RPDs had to be less than $20 \%$.

${ }^{\mathrm{B}}$ Analyte is present at a concentration above the MDL but less than the EQL.

\subsubsection{Analysis of Anions in TCLP Leachate}

Results from TCLP leachate analyses have been given in Table 10. The following conclusions can be made relative to the TCLP leachate anion analyses:

-The MCL was exceeded for nitrate plus nitrite (as nitrogen) by more than a factor of one hundred.

-The MCL was exceeded for sulfate by approximately a factor of two.

-Fluoride results were elevated due to interference from acetate in the TCLP leachate.

-No bromide, chloride, nitrite, or phosphate, were detected in the TCLP leachates.

Nitrate and sulfate concentrations exceeded the MCL. The nitrate concentrations were more than one hundred times the MCL for nitrate plus nitrite. The MCL for nitrate plus nitrite has been given in terms of nitrogen. Sulfate concentrations were approximately two times the MCL. These analyses met all QC specifications of SW-846 including requirements for matrix spike and laboratory recoveries, and precision indicators (RPD or RSD calculations).

Bromide, chloride, nitrite, and phosphate were not detected in any TCLP leachate samples. Chloride MDLs were low enough to show chloride concentrations were below the MCL. Neither bromide nor phosphate was a COPC, but these analytes are commonly reported with the other inorganic anions. Results could not be used to determine whether the TCLP leachate fluoride concentrations were below the MCL. 
Table 10. TCLP Leachate Anion Concentrations, MDLs, and EQLs

\begin{tabular}{|c||c||c|c||c|c|c|c|}
\hline \multicolumn{1}{|c||}{} & \multicolumn{1}{|c||}{ MCL (mg/L) } & \multicolumn{2}{c||}{ Sample Limits (mg/L) } & \multicolumn{5}{c|}{ Sample Results (mg/L) } \\
\hline- & - & ${ }^{1}$ MDL & ${ }^{1}$ EQL & Sample 1 & Sample 2 & Sample 3 & Average \\
\hline \hline Date & - & - & - & $9 / 29 / 03$ & $9 / 29 / 03$ & $9 / 29 / 03$ & - \\
\hline SRS ID & - & - & - & $41-\mathrm{TM} 1$ & $41-\mathrm{TM} 2$ & $41-\mathrm{TM} 3$ & - \\
\hline BWXS ID & - & - & - & $0310002-07 \mathrm{~A}$ & $0310002-08 \mathrm{~A}$ & $0310002-09 \mathrm{~A}$ & - \\
\hline \hline Bromide & - & 95 & $2.5 \times 10^{2}$ & $<2.5 \times 10^{2}$ & $<2.5 \times 10^{2}$ & $<2.5 \times 10^{2}$ & $<2.5 \times 10^{2}$ \\
\hline Chloride & $2.5 \times 10^{2}$ & 25 & $2.5 \times 10^{2}$ & $<2.5 \times 10^{2}$ & $<2.5 \times 10^{2}$ & $<2.5 \times 10^{2}$ & $<2.5 \times 10^{2}$ \\
\hline Fluoride & 4 & 12 & $2.5 \times 10^{2}$ & $\mathrm{Y}_{4} 4.88 \times 10^{2}$ & $\mathrm{Y}_{4.67 \times 10^{2}}$ & $\mathrm{Y}_{4} 4.92 \times 10^{2}$ & ${ }^{\mathrm{Y}} 4.82 \pm 0.13 \times 10^{2}$ \\
\hline Nitrate & - & 39 & $2.5 \times 10^{2}$ & $5.15 \times 10^{3}$ & $5.35 \times 10^{3}$ & $5.37 \times 10^{3}$ & $5.29 \pm 0.12 \times 10^{3}$ \\
\hline Nitrite & - & 52 & $2.5 \times 10^{2}$ & $<2.5 \times 10^{2}$ & $<2.5 \times 10^{2}$ & $<2.5 \times 10^{2}$ & $<2.5 \times 10^{2}$ \\
\hline Zitrogen & 10 & - & - & $1.163 \times 10^{3}$ & $1.209 \times 10^{3}$ & $1.213 \times 10^{3}$ & $1.19 \pm 0.027 \times 10^{3}$ \\
\hline Phosphate & - & $1.4 \times 10^{2}$ & $2.5 \times 10^{2}$ & $<2.5 \times 10^{2}$ & $<2.5 \times 10^{2}$ & $<2.5 \times 10^{2}$ & $<2.5 \times 10^{2}$ \\
\hline Sulfate & $2.5 \times 10^{2}$ & 41 & $2.5 \times 10^{2}$ & $5.07 \times 10^{2}$ & $5.31 \times 10^{2}$ & $4.48 \times 10^{2}$ & $5.0 \pm 0.43 \times 10^{2}$ \\
\hline
\end{tabular}

- Indicates a location in the table for which an entry would not be appropriate.

${ }^{1}$ MDLs from USEPA SW-846 studies in water. EQLs were the low calibration concentration.

${ }^{Y}$ Elevated fluoride concentrations due to interference with acetate from one component (acetic acid) of the TCLP leachate.

${ }^{\mathrm{Z}}$ Nitrate + nitrite as nitrogen. Since nitrite was not detected, nitrogen calculation from nitrate. [nitrogen] $=$ [nitrate] $/ 4.43$.

\subsubsection{Analysis of Anions in Tank 41 Saltstone}

Results have been given in Table 11 for the total anion concentrations in the Tank 41 Saltstone samples. These concentrations were determined using a USEPA Method 300 preparation followed by a USEPA SW-846 Method 9056 (ion chromatography) analysis. Results from the grout sample analyses were consistent with results from the TCLP leachate analyses. Nitrate plus nitrite concentrations were more than one hundred times the MCL in terms of nitrogen concentration from these two analytes. Sulfate concentrations were about two times the MCL for two samples. The third sulfate result appeared to be biased low for unknown reasons and was not used in calculating the average. Fluoride concentrations were shown to be approximately one fourth the MCL.

Table 11. Tank 41 Saltstone Total Anion Concentrations and EQLs

\begin{tabular}{|c|c|c|c|c|c|c|c|c|}
\hline - & MCL $(\mathrm{mg} / \mathbf{k g})$ & \multicolumn{2}{|c|}{ Sample $1(\mathrm{mg} / \mathrm{kg})$} & \multicolumn{2}{|c|}{ Sample $2(\mathrm{mg} / \mathrm{kg})$} & \multicolumn{2}{|c|}{ Sample $3(\mathrm{mg} / \mathrm{kg})$} & Average (mg/kg) \\
\hline Date & - & \multicolumn{2}{|c|}{ 9/29/03 } & \multicolumn{2}{|c|}{$9 / 29 / 03$} & \multicolumn{2}{|c|}{ 9/29/03 } & - \\
\hline SRS ID & - & \multicolumn{2}{|c|}{ 41-TM1 } & \multicolumn{2}{|c|}{ 41-TM2 } & \multicolumn{2}{|c|}{ 41-TM3 } & - \\
\hline BWXS ID & - & \multicolumn{2}{|c|}{$0310002-07 \mathrm{~A}$} & \multicolumn{2}{|c|}{$0310002-08 \mathrm{~A}$} & \multicolumn{2}{|c|}{$0310002-09 \mathrm{~A}$} & - \\
\hline- & - & $\overline{E Q L}$ & Result & EQL & Result & $\overline{\mathrm{EQL}}$ & Result & - \\
\hline Bromide & - & 22 & 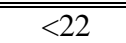 & 21 & 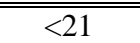 & 23 & $\frac{23}{<<23}$ & $\overline{c<21}$ \\
\hline Chloride & $5 \times 10^{3}$ & 22 & $<22$ & 21 & $<21$ & 23 & $<23$ & $<21$ \\
\hline Fluoride & 80 & 22 & $\mathrm{~J}_{21}$ & 21 & $<21$ & 23 & $<23$ & $J_{21}$ \\
\hline Nitrate & - & $4.35 \times 10^{3}$ & $1.11 \times 10^{5}$ & $4.2 \times 10^{3}$ & $1.12 \times 10^{5}$ & $2.3 \times 10^{3}$ & $8.5 \times 10^{4}$ & $1.1 \pm 0.18 \times 10^{5}$ \\
\hline Nitrite & - & $2.2 \times 10^{2}$ & $4.25 \times 10^{3}$ & $2.1 \times 10^{2}$ & $4.12 \times 10^{3}$ & $2.3 \times 10^{2}$ & $3.12 \times 10^{3}$ & $3.8 \pm 0.62 \times 10^{3}$ \\
\hline${ }^{\mathrm{Z}}$ Nitrogen & $2 \times 10^{2}$ & - & $2.64 \times 10^{4}$ & - & $2.81 \times 10^{4}$ & - & $2.02 \times 10^{4}$ & $2.5 \pm 0.41 \times 10^{4}$ \\
\hline Phosphate & - & 22 & $<22$ & 21 & $<21$ & 23 & $<23$ & $<21$ \\
\hline Sulfate & $5 \times 10^{3}$ & $2.2 \times 10^{3}$ & $9.5 \times 10^{3}$ & $2.1 \times 10^{3}$ & $1.02 \times 10^{4}$ & $2.3 \times 10^{3}$ & $\mathrm{Y}_{3.83 \times 10^{3}}$ & $9.9 \pm 0.69 \times 10^{3}$ \\
\hline
\end{tabular}

- Indicates a location in the table for which an entry would not be appropriate.

${ }^{\mathrm{J}}$ Fluoride was present in this sample at a concentration above the MDL but less than the EQL.

${ }^{\mathrm{Y}}$ This sulfate result was not used in determining an average or RSD. It appears to have been biased low.

${ }^{\mathrm{Z}}$ Nitrate + nitrite as nitrogen. Nitrogen calculated from nitrate and nitrite. [nitrogen] $=([$ nitrate $] / 4.43)+([$ nitrite $] / 3.28)$. 


\subsubsection{Quality Assurance for Anion Analyses}

Results from the matrix spike, laboratory control samples, blank analyses, and precision indicators have been given in Table 12. Anion analyses met all quality control specifications. The TCLP extraction blank fluoride concentration was similar to sample results. For sulfate, two sample results were used to determine a RPD for the grout result. The third result appeared to be errant, and was not used in determining the average sulfate concentration.

Table 12. Tank 41 Saltstone Anion Concentrations and EQLs

\begin{tabular}{|c|c|c|c|c|c|c|c|}
\hline- & \multicolumn{4}{|c|}{$\begin{array}{r}\text { TCLP Leachate } \\
\end{array}$} & \multicolumn{3}{|c|}{$\begin{array}{c}\text { Total Grout Sample } \\
\end{array}$} \\
\hline- & & overy $(\%)$ & RPD (\%) & Blank (mg/L) & Recovery (\%) & RSD (\%) & Blank (mg/kg) \\
\hline- & MS & Lab Control & - & - & Lab Control & - & - \\
\hline Bromide & 86 & 89 & - & $<2.5 \times 10^{2}$ & 95 & - & $\angle 2.5$ \\
\hline Chloride & 92 & 93 & - & $<2.5 \times 10^{2}$ & 96 & - & $<2.5$ \\
\hline Fluoride & 86 & 88 & 1.2 & $5.09 \times 10^{2}$ & 100 & - & $<2.5$ \\
\hline Nitrate & 75 & 92 & 1.3 & $<2.5 \times 10^{2}$ & 93 & 17 & $<2.5$ \\
\hline Nitrite & 98 & 94 & 8.9 & $<2.5 \times 10^{2}$ & 95 & 16 & $<2.5$ \\
\hline Phosphate & 90 & 91 & - & $<2.5 \times 10^{2}$ & 95 & - & $<2.5$ \\
\hline Sulfate & 90 & 93 & 7.0 & $<2.5 \times 10^{2}$ & 96 & $\mathrm{Z}_{7.0}$ & $<2.5$ \\
\hline
\end{tabular}

- Indicates a location in the table for which an entry would not be appropriate.

${ }^{\mathrm{Z}}$ This value is the RPD between the two sulfate results that were used to determine the average concentration in Table 2.

\subsection{Organic Compounds}

Toxicity Characteristic Leaching Procedure (TCLP) leachate organic analyte concentrations from the Tank 41 Saltstone waste form have been presented in this section. These analyses showed none of the COPCs was present above the MCLs. Toluene was detected at a very low concentration in one sample (at a concentration less than the USEPA SW-846 established MDL). Although a very low concentration of benzene was detected in one sample, benzene was also detected in the extraction blank. Application of the CLP 5x rule would identify the benzene results as a "nondetect".

\subsubsection{Analysis of Organic Compounds in TCLP Leachate}

Results from TCLP leachate analyses have been given in Table 13. No organic analyte result was higher than the MCLs. Toluene was detected in one TCLP leachate sample at a concentration less than the EQL. For analytes not detected, the EQL has been given in Table preceded by a "<". Benzene "nondetects" have been presented as " $<0.010$ ". Although the benzene EQL was not less than the MCL, the benzene MDL was less than the MCL.

\subsubsection{Quality Assurance for Analysis of Organic Compounds in TCLP Leachate}

Results from the matrix spike, matrix spike duplicate, laboratory control samples, and extraction blank analyses have been given in Table 14. Organic compound analyses met all quality control specifications except for the toluene matrix spike duplicate result. The toluene matrix spike duplicate recovery and the associated RPD were outside of acceptance 
ranges. This failure was indicative of a problem that was encountered in the purge and trap autosampler and was not indicative of poor quality in the sample data.

Table 13. TCLP Leachate Organic Compound Concentrations, MDLs, and EQLs

\begin{tabular}{|c||c|c||c|c||c|c|c|c|}
\hline \multicolumn{1}{|c||}{} & \multicolumn{2}{|c||}{ Regulatory Limits (mg/L) } & \multicolumn{2}{c||}{ Sample Limits (mg/L) } & \multicolumn{4}{c|}{ Sample Results (mg/L) } \\
\hline- & MCL & PRG & ${ }^{1}$ MDL & ${ }^{1}$ EQL & Sample 1 & Sample 2 & Sample 3 & Average \\
\hline \hline Date & - & - & - & - & $9 / 29 / 03$ & $9 / 29 / 03$ & $9 / 29 / 03$ & - \\
\hline \hline Benzene & $5.0 \times 10^{-3}$ & - & $1.0 \times 10^{-3}$ & 0.010 & $<0.010$ & ${ }^{\mathrm{JB}} 2.9 \times 10^{-3}$ & $<0.010$ & ${ }^{\mathrm{JB}} 2.9 \times 10^{-3}$ \\
\hline n-Butanol & - & 3.6 & 0.1 & 0.10 & $<0.10$ & $<0.10$ & $<0.10$ & $<0.10$ \\
\hline Toluene & 1.0 & - & $2.0 \times 10^{-3}$ & 0.010 & $\mathrm{Z}^{\mathrm{J}} 1.2 \times 10^{-3}$ & $<0.010$ & $<0.010$ & $\mathrm{Z}^{\mathrm{Z}} 1.2 \times 10^{-3}$ \\
\hline \hline Phenol & - & 22 & 0.05 & 1.0 & $<1.0$ & $<1.0$ & $<1.0$ & $<1.0$ \\
\hline
\end{tabular}

- Indicates a location in the table for which an entry would not be appropriate.

${ }^{1}$ MDLs from USEPA SW-846 studies in water. EQLs were low calibration concentration.

${ }^{\mathrm{J}}$ Analyte is present at a concentration above the MDL but less than the EQL.

B Application of the CLP 5x rule to this value would indicate that the sample result should be considered a "nondetect".

${ }^{\mathrm{Z}}$ Although a toluene peak was present in one sample, the concentration was less than the USEPA SW-846 established MDL.

Table 14. Organic Compound Matrix Spikes, Laboratory Control Samples, and Blanks

\begin{tabular}{|c||c|c|c||c|c|c|c||c|}
\hline \multicolumn{1}{|c||}{} & \multicolumn{3}{c||}{ Matrix Spikes } & \multicolumn{4}{c||}{ Laboratory Control Samples } & Blank (mg/kg) \\
\hline- & \multicolumn{1}{|c|}{ Recovery (\%) } & \multicolumn{1}{|c|}{ RPD (\%) } & \multicolumn{4}{c||}{ Recovery (\%) } & - \\
\hline- & MS & MSD & - & LCS31 & LCS51 & LCS52 & LCS54 & - \\
\hline \hline Benzene & 96 & 95 & 1 & - & 112 & 84 & 86 & $J^{2.2 \times 10^{-3}}$ \\
\hline n-Butanol & 87 & 96 & 10 & - & 84 & 95 & 105 & $<0.10$ \\
\hline Toluene & 28 & 105 & 116 & - & 125 & 84 & 86 & $<0.010$ \\
\hline \hline Phenol & 42 & 36 & 15 & 36 & - & - & - & $<1.0$ \\
\hline
\end{tabular}

- Indicates a location in the table for which an entry would not be appropriate.

J Analyte was present at a concentration above the MDL but less than the EQL.

\subsection{Radionuclides}

Toxicity Characteristic Leaching Procedure (TCLP) leachate radionuclide results have been presented in Table 15. The following conclusions can be made relative to these analyses:

-The gross alpha results exceeded the MCL by more than a factor of one thousand. -Radium isotopes could not be determined to the MCL because of elevated background. -Most beta and gamma activity was from cesium-137 and its daughter barium-137m. -Most of the alpha activity was from plutonium-238 and curium-244.

Matrix spike and laboratory control sample recoveries, RPD measurements, and blank results have been reported in the data package for these analyses. These QC indicators were acceptable except promethium-147 laboratory control sample recovery and blank results which suggested the reported promethium-147 results could be significantly higher than the true concentration. Because some Sample 2 results appeared to be biased low, only Sample 1 and Sample 3 results were used to calculate averages for the uranium isotopes. The high curium-244 concentrations appear to have been due to contamination from the SRTC shielded cells since the measured curium-244 concentration was higher than the concentration that would have been present in the TCLP leachate assuming curium-244 in the Tank 41 salt solution ${ }^{10}$ was completely leached from the Tank 41 Saltstone sample. 
Table 15. TCLP Leachate Radionuclide Concentrations and MDAs

\begin{tabular}{|c|c|c|c|c|c|c|c|c|}
\hline- & MCL (pCi/L) & \multicolumn{2}{|c|}{ Sample 1 (pCi/L) } & \multicolumn{2}{|c|}{ Sample $2(\mathrm{pCi} / \mathrm{L})$} & \multicolumn{2}{|c|}{ Sample $3(\mathrm{pCi} / \mathrm{L})$} & Ave (pCi/L) \\
\hline Date & - & \multicolumn{2}{|c|}{ 9/29/03 } & \multicolumn{2}{|c|}{ 9/29/03 } & \multicolumn{2}{|c|}{$9 / 29 / 03$} & - \\
\hline SRS ID & - & \multicolumn{2}{|c|}{ 41-TR1 } & \multicolumn{2}{|c|}{$41-\mathrm{TR} 2$} & \multicolumn{2}{|c|}{ 41-TR3 } & - \\
\hline BWXS ID & - & \multicolumn{2}{|c|}{$0310002-17$} & \multicolumn{2}{|c|}{$0310002-18$} & \multicolumn{2}{|c|}{$0310002-19$} & - \\
\hline- & 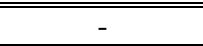 & ${ }^{\mathrm{I}} \mathrm{MDA}$ & Result & ${ }^{1}$ MDA & Result & ${ }^{\mathrm{T}} \mathrm{MDA}$ & Result & - \\
\hline H-3 & - & $1.3 \times 10^{4}$ & $1.7 \times 10^{4}$ & $1.3 \times 10^{4}$ & $<<1.3 \times 10^{4}$ & $1.3 \times 10^{4}$ & $<<1.3 \times 10^{4}$ & $1.7 \times 10^{4}$ \\
\hline Sr-90 & - & $5.7 \times 10^{4}$ & $9.1 \times 10^{5}$ & $6.8 \times 10^{4}$ & $4.5 \times 10^{5}$ & $5.8 \times 10^{4}$ & $1.1 \times 10^{6}$ & $8.1 \pm 3.2 \times 10^{5}$ \\
\hline Tc-99 & - & $8.8 \times 10^{2}$ & $7.8 \times 10^{4}$ & $1.5 \times 10^{3}$ & $7.3 \times 10^{4}$ & $9.6 \times 10^{2}$ & $1.0 \times 10^{5}$ & $8.4 \pm 1.6 \times 10^{4}$ \\
\hline U-232 & - & 83 & $<83$ & 1.1 & $<1.1$ & $3.7 \times 10^{2}$ & $<3.7 \times 10^{2}$ & $<83$ \\
\hline U-234 & - & 58 & $2.6 \times 10^{3}$ & 1.7 & 26 & $2.9 \times 10^{2}$ & $8.0 \times 10^{2}$ & $1.7 \pm 0.89 \times 10^{3}$ \\
\hline U-235 & - & 48 & $2.4 \times 10^{2}$ & 1.1 & 3.2 & $2.4 \times 10^{2}$ & $<2.4 \times 10^{2}$ & $2.4 \times 10^{2}$ \\
\hline U-236 & - & 48 & $Z_{<1.1 \times 10^{2}}$ & 1.1 & $\mathrm{Z}_{<1.9}$ & $2.4 \times 10^{2}$ & $<2.4 \times 10^{2}$ & $<1.1 \times 10^{2}$ \\
\hline U-238 & - & 48 & 80 & 1.1 & 1.9 & $2.4 \times 10^{2}$ & $<2.4 \times 10^{2}$ & 80 \\
\hline Pu-238 & - & 12 & $1.3 \times 10^{4}$ & 0.87 & $2.3 \times 10^{3}$ & 47 & $6.6 \times 10^{3}$ & $7.3 \pm 5.4 \times 10^{3}$ \\
\hline $\mathrm{Pu}-239 / 240$ & - & 12 & $5.9 \times 10^{2}$ & 0.69 & $1.1 \times 10^{2}$ & 47 & $2.2 \times 10^{2}$ & $3.1 \pm 2.6 \times 10^{2}$ \\
\hline Pu-241 & - & $1.7 \times 10^{3}$ & $6.1 \times 10^{3}$ & 75 & $6.5 \times 10^{2}$ & $8.8 \times 10^{3}$ & $<8.8 \times 10^{3}$ & $3.4 \pm 2.7 \times 10^{3}$ \\
\hline Am-241 & - & 8.9 & $7.2 \times 10^{2}$ & 0.57 & $1.4 \times 10^{2}$ & 65 & $3.6 \times 10^{2}$ & $4.1 \pm 2.9 \times 10^{2}$ \\
\hline Cm-242 & - & 10 & 11 & 0.58 & 1.6 & 62 & $<61$ & $6.5 \pm 4.9$ \\
\hline Cm-244 & - & 8.9 & $1.2 \times 10^{4}$ & 0.38 & $3.5 \times 10^{3}$ & 38 & $4.2 \times 10^{3}$ & $6.6 \pm 4.8 \times 10^{3}$ \\
\hline Gross • & 15 & $2.6 \times 10^{3}$ & $3.1 \times 10^{4}$ & $2.1 \times 10^{3}$ & $6.1 \times 10^{3}$ & $1.7 \times 10^{3}$ & $1.7 \times 10^{4}$ & $1.8 \pm 1.2 \times 10^{4}$ \\
\hline Gross • & - & $1.1 \times 10^{3}$ & $6.26 \times 10^{8}$ & $1.1 \times 10^{3}$ & $5.87 \times 10^{8}$ & $1.8 \times 10^{3}$ & $6.54 \times 10^{8}$ & $6.2 \pm 0.34 \times 10^{8}$ \\
\hline Co-60 & - & $7.2 \times 10^{3}$ & $<7.2 \times 10^{3}$ & $6.2 \times 10^{3}$ & $<6.2 \times 10^{3}$ & $6.3 \times 10^{3}$ & $<6.3 \times 10^{3}$ & $<6.2 \times 10^{3}$ \\
\hline Ru-106 & - & $1.4 \times 10^{6}$ & $<1.4 \times 10^{6}$ & $1.4 \times 10^{6}$ & $<1.4 \times 10^{6}$ & $1.3 \times 10^{6}$ & $<1.3 \times 10^{6}$ & $<1.3 \times 10^{6}$ \\
\hline Sb-125 & - & $7.0 \times 10^{5}$ & $<7.0 \times 10^{5}$ & $6.8 \times 10^{5}$ & $<6.8 \times 10^{5}$ & $6.6 \times 10^{5}$ & $<6.6 \times 10^{5}$ & $<6.6 \times 10^{5}$ \\
\hline Cs-137 & - & $2.1 \times 10^{5}$ & $7.60 \times 10^{8}$ & $2.1 \times 10^{5}$ & $7.2 \times 10^{8}$ & $2.0 \times 10^{5}$ & $6.69 \times 10^{8}$ & $7.2 \pm 0.46 \times 10^{8}$ \\
\hline Eu-154 & - & $2.1 \times 10^{4}$ & $<2.1 \times 10^{4}$ & $2.1 \times 10^{4}$ & $2.3 \times 10^{4}$ & $2.1 \times 10^{4}$ & $<2.1 \times 10^{4}$ & $<2.1 \times 10^{4}$ \\
\hline Ra-226 & 5 & $4.2 \times 10^{4}$ & $<4.2 \times 10^{4}$ & $4.6 \times 10^{4}$ & $<4.6 \times 10^{4}$ & $4.6 \times 10^{4}$ & $<4.6 \times 10^{4}$ & $<4.2 \times 10^{4}$ \\
\hline Ra-228 & 5 & $3.8 \times 10^{4}$ & $<3.8 \times 10^{4}$ & $3.8 \times 10^{4}$ & $<3.8 \times 10^{4}$ & $3.5 \times 10^{4}$ & $<3.5 \times 10^{4}$ & $<3.5 \times 10^{4}$ \\
\hline Pm-147 & - & $1.6 \times 10^{3}$ & $\mathrm{Z}_{<1.9 \times 10^{5}}$ & $2.0 \times 10^{3}$ & $Z_{<}<2.4 \times 10^{5}$ & $1.3 \times 10^{3}$ & $Z_{<}<3.2 \times 10^{5}$ & $\mathrm{Z}_{<1.9 \times 10^{5}}$ \\
\hline
\end{tabular}

- Indicates a location in the table for which an entry would not be appropriate.

${ }^{1}$ MDAs from standard practices based on counting statistics.

${ }^{\mathrm{Z}}$ Although the reported activity was greater than the MDA, evaluation of the spectra showed no photopeak to be present.

\subsection{CONCLUSIONS}

Analyses were successfully performed on the Tank 41 grout samples. Because some QC failures occurred during the metal analyte determinations and because the TCLP leachate interfered with fluoride analyses, the metals and anion concentrations were also determined on the grout samples. SCDHEC forms and a copy of the chain of custody have been included as Appendix A.

\subsection{Metals}

Preparation of the Tank 41H Saltstone samples and the subsequent TCLP analyses showed

-TCLP metal results were below SCDHEC MCLs except arsenic, lead, and selenium.

-TCLP leachate lithium concentrations were close to but slightly above the SCDHEC PRG.

-The laboratory MDL for antimony was higher than the MCL for this analyte.

-Results met all USEPA SW-846 QC specifications except aluminum and iron recoveries. 
The TCLP leachate concentrations were less than MCLs in SCDHEC Regulations R.61107.16, Subpart A, 16.4 except for arsenic, selenium, and lead. Arsenic results were approximately two times the MCL, and selenium concentrations were approximately three times the MCL. Although these results were above the MCL, they were less than ten times the MCL and therefore, arsenic and selenium results would not mandate disposal of the Tank 41 Saltstone in a Class 2 or 3 ISWLF. Because of instrumentation difficulties, the more sensitive atomic absorption analyses could not be completed. These analyses are being performed, and the associated data package is expected to be received at SRS by March 1, 2004. Results will be communicated to Dennis Conrad or a designee; however, the current intent is not to revise this document unless these results are higher than the values given here.

No lead was detected in the TCLP leachate; however, these measurements were not sensitive enough to measure to below the MCL. The TCLP measurements were sensitive enough to show that leachate concentrations were less than 10 times the MCL. This was sufficient to show a Class 2 or 3 ISWLF would not be required by the lead result. Total lead concentrations in the Tank 41 Saltstone showed that if all of the lead were to be leached from the grout sample, the TCLP leachate would exceed the MCL by a factor of close to twenty.

The TCLP leachate concentrations were less than PRGs given in Reference 6 except for lithium. Lithium results were slightly (less than six percent) above the lithium PRG. In addition, the lithium post spike was high (134\%). Since matrix spike and matrix spike duplicate recoveries (113.9\% and $114.7 \%$ respectively) were acceptable, the post spike did not constitute a QC failure but suggested a potential high bias in lithium results.

Analyses met all USEPA SW-846 quality assurance requirements except the matrix spike and matrix spike duplicate recoveries for aluminum and iron. Because the aluminum and iron results were well below the MCLs and because results from other COPCs mandated the more restrictive ISWLF classes, the total concentrations of these COPCs were not determined on the Tank 41 Saltstone samples.

\subsection{Anions}

Preparation of the Tank 41H Saltstone samples and the subsequent anion analyses showed

-Nitrate concentrations were one hundred times the SCDHEC MCL for nitrate plus nitrite. -Sulfate concentrations were greater than the SCDHEC MCL for sulfate.

-Chloride and fluoride were shown to be below SCDHEC MCLs.

-Bromide, chloride, and phosphate were not detected in any samples.

-Anions analyses met all QC specifications.

Anion concentrations were less than MCLs in SCDHEC Regulations R.61-107.16, Subpart A, 16.4 except for nitrate and sulfate. Nitrate results were slightly more than one hundred times the MCL, and sulfate results were about two times the MCLs. Because nitrate values were more than one hundred times the MCL, these results would mandate a Class 3 ISWLF. SCDHEC forms and a copy of the chain of custody have been included as Appendix A. 
Chloride was not detected in any sample. Because of acetate interference, TCLP leachate results could not be used to determine fluoride at concentrations low enough to evaluate compliance with the MCLs. Fluoride was detected in one of the samples analyzed for total fluoride in the Tank 41 grout. This result indicated that if all fluoride were released during TCLP analyses, the leachate fluoride concentration would be about one fourth the MCL.

\subsection{Organic Compounds}

Concentrations of all organic COPCs were lower than the SCDHEC MCLs. Toluene was detected in one of the samples but at about one one-thousandth the MCL. The toluene MSD analysis was hindered by a purge and trap autosampler problem. As a result the toluene MSD recovery and the RPD were outside acceptance ranges. All other quality control specifications were met for the organic compound analyses. Benzene was detected but was categorized as a "nondetect" after applying the CLP 5x rule.

\subsection{Radionuclides}

Preparation of the Tank 41H Saltstone samples and the subsequent radionuclide analyses showed

-Gross alpha concentrations were one thousand times the SCDHEC MCL for gross alpha. -Radium isotopes were not determined to the SCDHEC MCL.

-Quality control indicators were indicative of quality results except for promethium-147.

Gross alpha results were more than one thousand times the MCL in SCDHEC Regulations R.61-107.16, Subpart A, 16.4 for gross alpha. Because gross alpha values were more than thirty times the MCL, these results would mandate a Class 3 ISWLF. Because the plutonium-238 results exceeded thirty times the MCL for total alpha-emitting radionuclides, the suspected curium-244 contamination of the samples (most likely from the SRTC shielded cells facility) did not impact the disposal facility classification. Radium isotopes were not determined to activities low enough to determine whether the TCLP leachate concentrations were below the MCL for these radionuclides.

\subsection{SUMMARY}

The most significant results from characterization of the Tank 41H 28-day cure Saltstone were

-TCLP leachate nitrate and alpha results were high enough to require a Class 3 ISWLF. -Five COPC TCLP leachate results were between the MCLs and ten times the MCLs. -The Tank 41 Saltstone met requirements of a nonhazardous radioactive waste form. 
After curing for more than 28 days, the Tank $41 \mathrm{H}$ Saltstone produced with Z-Area premix material exceeded thirty times the nitrate and gross alpha MCLs given in SCDHEC Regulations R.61-107.16, Subpart A, 16.4 indicating the waste form would require disposal in a Class 3 ISWLF. The nitrate results were consistent with previously reported results from TCLP analyses of the Tank 50 Saltstone. The higher gross alpha results were consistent with the concentration of alpha-emitting radionuclides in the Tank $41 \mathrm{H}$ salt solution.

Arsenic, selenium, and sulfate results exceeded the MCLs but were less than ten times the MCLs, and lithium results were slightly higher than the PRG. In addition, lead concentrations in the TCLP leachate were shown to be less than the lead MCL but could not be shown to be less than the MCL. Arsenic, selenium, and lead concentrations were close to the method detection limits. Therefore, these results have a higher degree of uncertainty associated with them than results for other analytes.

Previously TCLP analyses for the primary eight Resource Conservation and Recovery Act (RCRA) metals showed that after a five-day cure, the Tank 41H Saltstone met the requirements of a nonhazardous radioactive waste form. ${ }^{2}$ Results from analysis of the 28-day Tank 41H Saltstone supported the previous analyses. Concentrations for the eight RCRA metals were similar for the two sets of analyses.

\subsection{REFERENCES}

1. WSRC Manual 1S, Procedure 4.01 “Acceptance Criteria for Aqueous Waste Sent to the Z Area Saltstone Production Facility” (Rev. 4, INTERIM, 03/13/03).

2. Alex Cozzi, Christine Langton, and Daro Ferrara, "Tank 41H Saltstone TCLP RCRA Metal Results," Westinghouse Savannah River Company Report Number WSRC-RP2003-00439, Rev. 0, September 23, 2003.

3. Christine A. Langton, "Technical Basis for LCS Saltstone Disposal Vault Classification", Westinghouse Savannah River Company Technical Report Number WSRC-TR-200300431, Rev. 0, September 2003.

4. United States Environmental Protection Agency, "Land Disposal Restrictions", Code of Federal Regulations 40CFR268.40, September 19, 1994.

5. South Carolina Department of Health and Environmental Control, SCDHEC Regulations R.61-107.16 Subpart A, 16.4.

6. C. A. Langton. "Task Technical and QA Plan: Tank 41/LCS Saltstone Processability and TCLP Testing," Westinghouse Savannah River Company Report Number WSRC-RP2003-00572, Rev. 0, August 28, 2003.

7. Office of Solid Waste, United States of America Environmental Protection Agency, "Test Methods for Evaluating Solid Waste, Physical/Chemical Methods," USEPA SW-846, Third Edition, EPA Publication Number 955-001-00000-1. 
8. Office of Research and Development, United States of America Environmental Protection Agency, "Methods for the Determination of Inorganic Substances in Environmental Samples," USEPA EPA/600/R-93-100, August 1993.

9. United States Environmental Protection Agency, "EPA Guidance for Definitive Data Applicable to the CERCLA (Superfund) Program".

10. C. J. Martino, W. R. Wilmarth, D. P. Diprete, and C. C. Diprete, "Tank 41H Dissolved Saltcake Sample (HTF-E-03-91 - 92) Saltstone Waste Acceptance Criteria Analysis," Westinghouse Savannah River Company Report Number WSRC-TR-2003-00380, Rev. 1, September 22, 2003. 
APPENDIX A. SCDHEC FORMS AND CHAIN OF CUSTODY 
WSRC-TR-2004-00051

Revision 0

\begin{tabular}{|c|c|c|c|c|c|c|c|c|c|c|c|}
\hline \multirow{4}{*}{\multicolumn{2}{|c|}{ 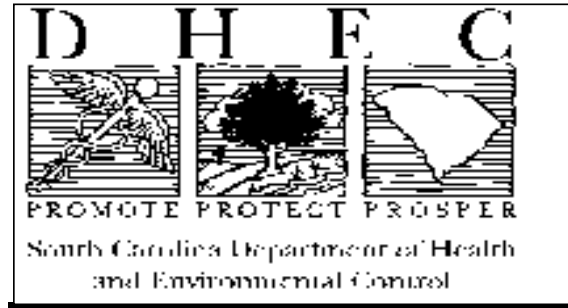 }} & \multirow{4}{*}{\multicolumn{2}{|c|}{$\begin{array}{l}\text { Type Data: } \\
\text { Company Name: } \\
\text { Subject/Project: }\end{array}$}} & \multicolumn{6}{|c|}{$\begin{array}{l}\text { Industrial Inorganic TCLP/R.61-58.5 } \\
\text { Westinghouse Savannah River Company } \\
\text { Low-Curie Salt }\end{array}$} & \multirow{2}{*}{$\begin{array}{l}\text { Date: } \\
\text { r Liter }\end{array}$} & \multirow[t]{2}{*}{$01 / 30 / 04$} \\
\hline & & & & & & & \multicolumn{3}{|c|}{ Results in Milligrams per Liter } & & \\
\hline & & & & & & & \multicolumn{3}{|c|}{ Waste Stream 1} & & \\
\hline & & & & & & & 9/29/2003 & 9/29/2003 & 9/29/2003 & - & - \\
\hline \multicolumn{7}{|c|}{ Facility Sample ID \# } & 41-TR1 & 41-TR2 & 41-TR3 & - & - \\
\hline \multicolumn{7}{|c|}{ Laboratory Sample ID \# } & $0310002-17$ & $0310002-18$ & $0310002-19$ & - & - \\
\hline \multicolumn{7}{|c|}{ Laboratory Name } & BWXS-NELS & BWXS-NELS & BWXS-NELS & - & - \\
\hline \multicolumn{7}{|c|}{ SC Laboratory Certification \# } & Pending & Pending & Pending & - & - \\
\hline \multicolumn{7}{|c|}{ Subcontracted Laboratory Certification \# } & $\ldots$ & $-\ldots$ & $-\ldots$ & - & - \\
\hline \multicolumn{7}{|c|}{ Subcontracted Laboratory Name } & $\ldots$ & $-\ldots$ & $\ldots$ & - & - \\
\hline \multicolumn{7}{|c|}{ Laboratory Receipt Information (Chain of Custody Must be Attached) } & Attached & Attached & Attached & - & - \\
\hline \multicolumn{7}{|c|}{$\begin{array}{c}\text { Inorganic TCLP Chemicals } \\
\end{array}$} & 1 & 1 & 1 & 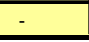 & - \\
\hline Analytical Parameter & $\begin{array}{l}\begin{array}{c}\text { Analytical } \\
\text { Method }\end{array} \\
\end{array}$ & \begin{tabular}{|l} 
Quantitation \\
Limit (mg/l) \\
\end{tabular} & $\operatorname{MCL}(\mathrm{mg} / \mathrm{l})$ & $\begin{array}{c}10 \times \mathrm{MCL} \\
(\mathrm{mg} / \mathrm{l})\end{array}$ & $\begin{array}{c}30 \times \mathrm{MCL} \\
(\mathrm{mg} / \mathrm{l})\end{array}$ & - & & & & & \\
\hline Antimony & $6010 \mathrm{~B}$ & 0.28 & 0.006 & 0.06 & 0.18 & - & $<0.028$ & $<0.028$ & $<0.028$ & - & - \\
\hline Arsenic & $6010 \mathrm{~B}$ & 0.12 & 0.01 & 0.10 & 0.3 & - & $<0.012$ & $0.021(\mathrm{~B})$ & $0.016(\mathrm{~B})$ & - & - \\
\hline Barium & $6010 \mathrm{~B}$ & 0.012 & 2 & 20.00 & 60 & - & 0.122 & 0.110 & 0.152 & - & - \\
\hline Beryllium & $6010 \mathrm{~B}$ & $1.70 \mathrm{E}-03$ & 0.004 & 0.04 & 0.12 & - & $<1.7 \mathrm{E}-4$ & $1.1 \mathrm{E}-03$ & $<1.7 \mathrm{E}-4$ & - & - \\
\hline Cadmium & $6010 \mathrm{~B}$ & 0.016 & 0.005 & 0.05 & 0.15 & - & $<1.6 \mathrm{E}-3$ & $2.78 \mathrm{E}-3(\mathrm{~B})$ & $2.11 \mathrm{E}-3(\mathrm{~B})$ & - & - \\
\hline Chloride & 9056 & 250 & 250 & 2500.00 & 7500 & - & $<250$ & $<250$ & $<250$ & - & - \\
\hline Chromium & $6010 B$ & 0.032 & 0.1 & 1.00 & 3 & - & $0.0268(\mathrm{~B})$ & $0.0276(\mathrm{~B})$ & $0.0274(\mathrm{~B})$ & - & - \\
\hline Copper & $6010 \mathrm{~B}$ & 0.059 & 1.3 & 13.00 & 39 & - & $<5.9 \mathrm{E}-3$ & $<5.9 \mathrm{E}-3$ & $<5.9 \mathrm{E}-3$ & - & - \\
\hline Cyanide (as free cyanide) & $9010 \mathrm{~B} / 9040^{3}$ & - & 0.2 & 2.00 & 6 & - & - & - & - & - & - \\
\hline Fluoride & 9056 & 250 & 4 & 40.00 & 120 & - & 488 & 467 & 492 & - & - \\
\hline Iron & $6010 \mathrm{~B}$ & 0.051 & 0.3 & 3.00 & 9 & - & $0.044(\mathrm{~B})$ & 0.071 & 0.098 & - & - \\
\hline Lead & $6010 \mathrm{~B}$ & 0.28 & 0.015 & 0.15 & 0.45 & - & $<0.028$ & $<0.028$ & $<0.028$ & - & - \\
\hline Manganese & $6010 \mathrm{~B}$ & 0.012 & 0.05 & 0.50 & 1.5 & - & $3.8 \mathrm{E}-3(\mathrm{~B})$ & $2.9 \mathrm{E}-3(\mathrm{~B})$ & 0.0127 & - & - \\
\hline Mercury & $7470 \mathrm{~A}$ & $1.40 \mathrm{E}-03$ & 0.002 & 0.02 & 0.06 & - & $<1.4 \mathrm{E}-4$ & $1.8 \mathrm{E}-4(\mathrm{~B})$ & $8.1 \mathrm{E}-4(\mathrm{~B})$ & - & - \\
\hline Nitrate (as Nitrogen) & 9056 & 56 & 10 & 100.00 & 300 & - & $1.163 \mathrm{E}+03$ & $1.209 \mathrm{E}+03$ & $1.213 \mathrm{E}+03$ & - & - \\
\hline Nitrite (as Nitrogen) & 9056 & 76 & 10 & 100.00 & 300 & - & $<76$ & $<76$ & $<76$ & - & - \\
\hline Nitrate/Nitrite (Total) & 9056 & 132 & 10 & 100.00 & 300 & - & $1.163 \mathrm{E}+03$ & 1.209E+03 & $1.213 \mathrm{E}+03$ & - & - \\
\hline Selenium & $6010 \mathrm{~B}$ & 0.28 & 0.05 & 0.50 & 1.5 & - & $0.156(\mathrm{~B})$ & $0.150(\mathrm{~B})$ & $0.163(\mathrm{~B})$ & - & - \\
\hline Silver & $6010 \mathrm{~B}$ & 0.054 & 0.1 & 1.00 & 3 & - & $<5.4 \mathrm{E}-3$ & $<5.4 \mathrm{E}-3$ & $<5.4 \mathrm{E}-3$ & - & - \\
\hline Sulfate & 9056 & 250 & 250 & 2500.00 & 7500 & - & 507 & 531 & 448 & - & - \\
\hline Thallium & 7841 & - & 0.002 & 0.02 & 0.06 & - & - & - & - & - & - \\
\hline Aluminum ${ }^{7}$ & $6010 \mathrm{~B}$ & 0.3 & 36 & $3.6 \mathrm{E}+02$ & 1080 & - & $0.30(\mathrm{~B})$ & $0.16(\mathrm{~B})$ & $0.75(\mathrm{~B})$ & - & - \\
\hline Boron $^{7}$ & $6010 \mathrm{~B}$ & 0.18 & 3.3 & $3.3 \mathrm{E}+01$ & 99 & - & 0.517 & 0.483 & 0.37 & - & - \\
\hline Cobalt & $6010 \mathrm{~B}$ & 0.026 & 2.2 & $2.2 \mathrm{E}+01$ & 66 & - & $<2.6 \mathrm{E}-3$ & $<2.6 \mathrm{E}-3$ & $<2.6 \mathrm{E}-3$ & - & - \\
\hline Lithium $^{7}$ & $6010 \mathrm{~B}$ & 0.11 & 0.73 & 7.30 & 21.9 & - & 0.763 & 0.766 & 0.710 & - & - \\
\hline Molybdenum $^{7}$ & $6010 \mathrm{~B}$ & 0.23 & 0.18 & 1.80 & 5.4 & - & 0.486 & 0.484 & 0.442 & - & - \\
\hline \begin{tabular}{|l|l} 
Nickel $^{7}$ \\
\end{tabular} & $6010 \mathrm{~B}$ & 0.069 & 0.73 & 7.30 & 21.9 & - & $<6.9 \mathrm{E}-3$ & $<6.9 \mathrm{E}-3$ & $<6.9 \mathrm{E}-3$ & - & - \\
\hline Strontium, stable & $6010 \mathrm{~B}$ & 0.013 & 22 & $2.2 \mathrm{E}+02$ & 660 & - & 0.957 & 0.912 & 1.02 & - & - \\
\hline Uranium $^{7}$ & Radionuclides ${ }^{8}$ & $1.0 \mathrm{E}-06$ & 7.3 & 73.00 & 219 & - & $3.5 \mathrm{E}-07$ & $7.2 \mathrm{E}-09$ & $8.2 \mathrm{E}-07$ & - & - \\
\hline
\end{tabular}


WSRC-TR-2004-00051

Revision 0

\begin{tabular}{|c|c|c|c|c|c|}
\hline Quality Assurance (for above samples) & & & - & & \\
\hline TCLP Batch \# & $683-05$ & $683-05$ & $683-05$ & - & - \\
\hline Digestion Batch \# & $640-88$ & $640-88$ & $640-88$ & - & - \\
\hline TCLP Extraction Batch \# & $683-05$ & $683-05$ & $683-05$ & - & ב- \\
\hline Method Blank & S683-05B1 & S683B5-01 & S683-05B1 & - & - \\
\hline Laboratory Control Sample (LCS) & PB MS 640-88 & PB MS $640-88$ & PB MS 640-88 & - & 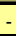 \\
\hline Matrix Spike (MS) & $0310002-19 \mathrm{MS}$ & $0310002-19 \mathrm{MS}$ & $0310002-19 \mathrm{MS}$ & - & - \\
\hline Matrix Spike Duplicate (MSD) & 0310002-19MSL & $0310002-19 \mathrm{MSL}$ & $0310002-19 \mathrm{MSC}$ & - & - \\
\hline LCS Recovery $(\%)$ & Acceptable & Acceptable & Acceptable & - & 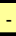 \\
\hline MS Recovery (\%) & $\mathrm{NA}(\mathrm{Al}, \mathrm{Fe})$ & $\mathrm{NA}(\mathrm{Al}, \mathrm{Fe})$ & $\mathrm{NA}(\mathrm{Al}, \mathrm{Fe})$ & - & - \\
\hline MSD Recovery (\%) & $\mathrm{NA}(\mathrm{Al}, \mathrm{Fe})$ & $\mathrm{NA}(\mathrm{Al}, \mathrm{Fe})$ & $\mathrm{NA}(\mathrm{Al}, \mathrm{Fe})$ & - & ב- \\
\hline \multicolumn{6}{|l|}{ Footnotes: } \\
\hline \multicolumn{6}{|l|}{ 1. Subcontracted Laboratory used for this Analyte. } \\
\hline \multicolumn{6}{|l|}{ 2. $6010 \mathrm{~B}$ can be used with Trace ICP. } \\
\hline \multicolumn{6}{|l|}{ 3. Spectrophotometric. } \\
\hline \multicolumn{6}{|l|}{ 4. Alternate Method 531.1. } \\
\hline \multicolumn{5}{|l|}{ Nuclear - EPA Methods. } & \\
\hline \multirow{2}{*}{\multicolumn{6}{|c|}{ Million of fibers per liter. }} \\
\hline & & & & & \\
\hline \multicolumn{6}{|l|}{ 3. To meet the PRG, individual uranium isotopes were measured. Uranium is being reported as the sum of the uranium isotopes. } \\
\hline & & & & & \\
\hline
\end{tabular}


WSRC-TR-2004-00051

Revision 0

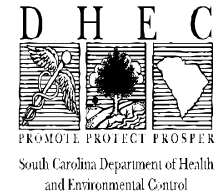

Date: $\quad 01 / 30 / 04$

\section{LABORATORY: \\ METHOD NAME:}

SUBJECT:
BWXS - NELS

SW-846-6010B, SW-846-9056, SW-846-7470A, and Alpha Counting

QA- Blk, Laboratory Control Sample (LCS), Matrix Spike (MS), Matrix Spike Duplicate (MSD)

Reference: Industrial Inorganic TCLP/R.61-58.5

Instrument: BWXT - Miscellaneous

\begin{tabular}{|c|c|c|c|c|c|c|c|c|c|c|c|c|c|c|c|c|c|}
\hline & \multicolumn{8}{|c|}{ Analyte Concentrations, $\mathrm{mg} / \mathrm{I}$} & \multicolumn{8}{|c|}{ Recovery Percent } & \multirow{2}{*}{$\begin{array}{c}\text { Flags } \\
-\end{array}$} \\
\hline Analytes & $\mathrm{RDL}$ & MDL & Blank & LCS & LCSD & MS & MSD & Other & LCS & LCSD & MS & MSD & Ave MS/MSD & REC Limits & $\%$ RPD & RPD Limits & \\
\hline Antimony & 0.006 & 0.028 & $<0.025$ & 1.00 & & 1.00 & 1.00 & - & 99.1 & - & 86.3 & 86.5 & 86.4 & $75-125$ & 1.7 & $10 \%$ & - \\
\hline Arsenic & 0.01 & 0.012 & $<0.011$ & 1.00 & - & 1.00 & 1.00 & - & 99.2 & - & 86.8 & 86.5 & 86.6 & $75-125$ & 0.3 & $10 \%$ & - \\
\hline Barium & 2 & 0.0012 & 0.025 & 1.00 & - & 1.00 & 1.00 & - & 97.6 & - & 76.2 & 75.5 & 75.8 & $75-125$ & 0.8 & $10 \%$ & - \\
\hline Beryllium & 0.004 & 0.00017 & $0.0002(\mathrm{~B})$ & 1.00 & - & 1.00 & 1.00 & - & 100.0 & - & 83.1 & 82.2 & 82.7 & $75-125$ & 1.1 & $10 \%$ & - \\
\hline Cadmium & 0.0050 & 0.0016 & $0.0016(\mathrm{~B})$ & 1.00 & - & 1.00 & 1.00 & - & 99.5 & - & 79.9 & 79.3 & 79.6 & $75-125$ & 0.8 & $10 \%$ & - \\
\hline Chloride & 250 & 25 & $<250$ & 2000 & - & 2000 & - & - & 93 & - & 92 & - & 92 & $75-125$ & $O(A)$ & $10 \%$ & - \\
\hline Chromium & 0.1 & 0.0032 & $0.004(\mathrm{~B})$ & 1.00 & - & 1.00 & 1.00 & - & 99.7 & - & 78.6 & 78.1 & 78.3 & $75-125$ & 0.6 & $10 \%$ & - \\
\hline Copper & 1.3 & 0.0059 & $0.020(\mathrm{~B})$ & 1.00 & - & 1.00 & 1.00 & - & 99.5 & - & 83.7 & 82.7 & 83 & $75-125$ & 1.1 & $10 \%$ & - \\
\hline $\begin{array}{c}\text { Cyanide } \\
\text { (as free cyanide) }\end{array}$ & 0.2 & - & - & - & - & - & - & - & - & - & - & - & - & - & - & - & - \\
\hline Fluoride & 4 & 12 & 509 & 2000 & - & 2000 & - & - & 88 & - & 86 & - & 86 & $75-125$ & $1.2(\mathrm{~A})$ & $10 \%$ & - \\
\hline Iron & 0.3 & 0.0051 & $0.045(\mathrm{~B})$ & 1.00 & - & 1.00 & 1.00 & - & 105.0 & - & 69.0 & 67.7 & 68 & $75-125$ & 1.7 & $10 \%$ & $\mathrm{~N}$ \\
\hline Lead & 0.015 & 0.028 & $<0.025$ & 1.00 & - & 1.00 & 1.00 & - & 100.0 & - & 76.8 & 78.5 & 78 & $75-125$ & 2.2 & $10 \%$ & - \\
\hline Manganese & 0.05 & 0.0012 & $0.006(\mathrm{~B})$ & 1.00 & - & 1.00 & 1.00 & - & 97.3 & - & 76.4 & 75.9 & 76.1 & $75-125$ & 0.6 & $10 \%$ & - \\
\hline Mercury & 0.002 & 0.00014 & $0.012(\mathrm{~B})$ & 0.00471 & - & 0.0050 & 0.0050 & - & 107.6 & - & 106.9 & 105.9 & 106 & $75-125$ & 0.9 & $10 \%$ & - \\
\hline $\begin{array}{c}\text { Nitrate } \\
\text { (as Nitrogen) }\end{array}$ & 10 & 9 & $<56$ & 452.00 & - & 452.00 & - & - & 92 & - & 75 & - & 75 & $75-125$ & $1.3(\mathrm{~A})$ & $10 \%$ & - \\
\hline $\begin{array}{c}\text { Nitrite } \\
\text { (as Nitrogen) }\end{array}$ & 10 & 16 & $<76$ & 610.00 & - & 610.00 & - & - & 94 & - & 98 & - & 98 & $75-125$ & $8.9(\mathrm{~A})$ & $10 \%$ & - \\
\hline $\begin{array}{l}\begin{array}{c}\text { Nitrate/Nitrite } \\
\text { (Total) }\end{array} \\
\end{array}$ & 10 & 16 & $<132$ & 1062.00 & - & 1062.00 & - & - & 93 & - & 77 & - & 77 & $75-125$ & $1.4(\mathrm{~A})$ & $10 \%$ & - \\
\hline Selenium & 0.05 & 0.028 & $<0.025$ & 1.00 & - & 1.00 & 1.00 & - & 98.5 & - & 90.4 & 88.5 & 89 & $75-125$ & 1.8 & $10 \%$ & - \\
\hline Silver & 0.1 & 0.0054 & $0.0052(\mathrm{~B})$ & 1.00 & - & 1.00 & 1.00 & - & 98.3 & - & 80.9 & 80.5 & 80.7 & $75-125$ & 0.5 & $10 \%$ & - \\
\hline Sulfate & 250 & 41 & \begin{tabular}{|l|}
$<250$ \\
\end{tabular} & 2000 & - & 2000 & - & - & 93.0 & - & 90 & - & 90 & $75-125$ & $7.0(\mathrm{~A})$ & $10 \%$ & - \\
\hline Thallium & 0.002 & - & - & - & - & - & - & - & - & - & - & - & - & - & - & - & - \\
\hline Aluminum & 36 & 0.03 & \begin{tabular}{|l|}
$0.097(\mathrm{~B})$ \\
\end{tabular} & 1.00 & - & 1.00 & 1.00 & - & 96.9 & - & 31.7 & 30.1 & 30.9 & $75-125$ & 1.7 & $10 \%$ & $\mathrm{~N}$ \\
\hline Boron & 3.3 & 0.018 & $0.12(\mathrm{~B})$ & 1.00 & - & 1.00 & 1.00 & - & 103.0 & - & 92.9 & 89.0 & 91 & $75-125$ & 3.1 & $10 \%$ & - \\
\hline Cobalt & 2.2 & 0.0026 & $<0.0023$ & 1.00 & - & 1.00 & 1.00 & - & 101.0 & - & 79.2 & 79.1 & 79.2 & $75-125$ & 0.2 & $10 \%$ & - \\
\hline Lithium & 0.73 & 0.011 & $<0.0097$ & 1.00 & - & 1.00 & 1.00 & - & 105.0 & - & 113.9 & 114.7 & 114.4 & $75-125$ & 0.4 & $10 \%$ & - \\
\hline Molybdenum & 0.18 & 0.023 & $<0.021$ & 1.00 & - & 1.00 & 1.00 & - & 103.0 & - & 96.7 & 93.6 & 95 & $75-125$ & 2.3 & $10 \%$ & - \\
\hline Nickel & 0.73 & 0.0069 & $<0.0062$ & 1.00 & - & 1.00 & 1.00 & - & 102.0 & - & 79.4 & 79.7 & 79.6 & $75-125$ & 0.3 & $10 \%$ & - \\
\hline Strontium, stable & 22 & 0.0013 & 0.014 & 1.00 & - & 1.00 & 1.00 & - & 97.6 & - & 97.9 & 97.0 & 97.5 & $75-125$ & 0.5 & $10 \%$ & - \\
\hline Uranium & 7.3 & 0.000001 & 3.E-08 & $4.70 \mathrm{E}-07$ & - & - & - & - & 126 & - & - & - & - & - & 13 & - & - \\
\hline
\end{tabular}

(A) These RPD were determined from duplicate analyses of the same sample. 
WSRC-TR-2004-00051

Revision 0

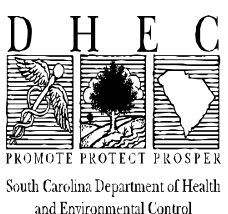

Sourt Carodina Department of Healts
and Envirommental Control

\section{Cross Reference Report for QA and Analytes}

Analytical Method Reference:

Lab Reference (to Facility Sample):

Subject / Project:

Facility:
SW846 3015 / 6010B - 7470A (TCLP Metals)

BWXS - NELS (SDG 0310002)

Low-Curie Salt

Westinghouse Savannah River Compan

\begin{tabular}{|c|c|c|c|c|c|c|}
\hline LAB ID \# & FACILITY SAMP ID \# & TC EXTR BATCH \# & DIGEST BATCH \# & ANALYSIS BATCH \# & OTHER & COMMENTS \\
\hline S683-05B1 (TCLP EXT BLANK) & None & $683-05$ & $640-88$ & A1108 / A1029A / A1031 & --- & 6010B ICP Metals \\
\hline PB (METHOD BLANK) & None & $683-05$ & $640-88$ & A1108 / A1029A / A1031 & --- & 6010B ICP Metals \\
\hline LCS P093-500 & None & $683-05$ & $640-88$ & A1108 / A1029A / A1031 & $-\cdots$ & 6010B ICP Metals \\
\hline 0310002-19AMS & 41-TR3 & $683-05$ & $640-88$ & A1108 / A1029A / A1031 & $-\cdots$ & 6010B ICP Metals \\
\hline 0310002-19AMSD & 41-TR3 & $683-05$ & $640-88$ & A1108 / A1029A / A1031 & --- & 6010B ICP Metals \\
\hline $0310002-17 \mathrm{~A}$ & 41-TR1 & $683-05$ & $640-88$ & $\mathrm{~A} 1108$ / A1029A / A1031 & -- & 6010B ICP Metals \\
\hline 0310002-18A (DUP) & 41-TR2 & $683-05$ & $640-88$ & A1108 / A1029A / A1031 & 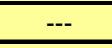 & 6010B ICP Metals \\
\hline $0310002-19 \mathrm{~A}$ & 41-TR3 & $683-05$ & $640-88$ & A1108 / A1029A / A1031 & -- & 6010B ICP Metals \\
\hline S683-05B1 (TCLP EXT BLANK) & None & $683-05$ & $605-45$ & 031022 & -- & 7470A CVAA HG \\
\hline 0 PPB (METHOD BLANK) & None & $683-05$ & $605-45$ & 031022 & $\overline{---}$ & 7470A CVAA HG \\
\hline LCS P093-500 & None & $683-05$ & $605-45$ & 031022 & $\begin{array}{c}-- \\
-\end{array}$ & 7470A CVAA HG \\
\hline 0310002-10AMS & 41-TM4 & $683-05$ & $605-45$ & 031022 & $-\cdots$ & 7470A CVAA HG \\
\hline 0310002-11AMSD & 41-TM5 & $683-05$ & $605-45$ & 031022 & $-\cdots$ & 7470A CVAA HG \\
\hline 0310002-07A & 41-TM1 & 683-05 & $605-45$ & 031022 & $\begin{array}{ll}-- \\
\end{array}$ & 7470A CVAA HG \\
\hline 0310002-08A (DUP) & 41-TM2 & $683-05$ & $605-45$ & 031022 & $-\cdots$ & 7470A CVAA HG \\
\hline $0310002-09 \mathrm{~A}$ & 41-TM3 & $683-05$ & $605-45$ & 031022 & --- & 7470A CVAA HG \\
\hline & & & & & & \\
\hline & & & & & & \\
\hline & & & & & & \\
\hline & & & & & & \\
\hline & & & & & & \\
\hline & & & & & & \\
\hline & & & & & & \\
\hline & & & & & & \\
\hline & & & & & & \\
\hline & & & & & & \\
\hline & & & & & & \\
\hline & & & & & & \\
\hline & & & & & & \\
\hline
\end{tabular}




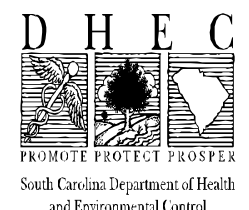

South Carolina Department of Health
and Environmental Confrol

\section{Cross Reference Report for QA and Analytes}

Analytical Method Reference:

Lab Reference (to Facility Sample):

Subject / Project:

Facility:
SW846 3015 / 6010B (Total Metals)

BWXS - NELS (SDG 0310002)

Low-Curie Salt

Westinghouse Savannah River Company

\begin{tabular}{|c|c|c|c|c|c|c|}
\hline LAB ID \# & FACILITY SAMP ID \# & TC EXTR BATCH \# & DIGEST BATCH \# & ANALYSIS BATCH \# & OTHER & COMMENTS \\
\hline PB (METHOD BLANK) & None & --- & $693-18$ & $\mathrm{~A} 1204$ & --- & 6010B ICP Metals \\
\hline PP D036 (LCS) & None & -- & 693-18 & A1204 & --- & 6010B ICP Metals \\
\hline 0310002-01AMS & 41-TE1 & --- & $693-18$ & A1204 & --- & 6010B ICP Metals \\
\hline 0310002-01AMSD & 41-TE1 & --- & $693-18$ & A1204 & --- & 6010B ICP Metals \\
\hline $0310002-01 \mathrm{~A}$ & 41-TE1 & --- & $693-18$ & A1204 & --- & 6010B ICP Metals \\
\hline 0310002-01A (DUP) & 41-TE1 & --- & $693-18$ & A1204 & --- & 6010B ICP Metals \\
\hline & & & & & & \\
\hline & & & & & & \\
\hline & & & & & & \\
\hline & & & & & & \\
\hline & & & & & & \\
\hline & & & & & & \\
\hline & & & & & & \\
\hline & & & & & & \\
\hline & & & & & & \\
\hline & & & & & & \\
\hline & & & & & & \\
\hline & & & & & & \\
\hline & & & & & & \\
\hline & & & & & & \\
\hline & & & & & & \\
\hline & & & & & & \\
\hline & & & & & & \\
\hline & & & & & & \\
\hline & & & & & & \\
\hline & & & & & & \\
\hline & & & & & & \\
\hline & & & & & & \\
\hline & & & & & & \\
\hline
\end{tabular}


WSRC-TR-2004-00051

Revision 0

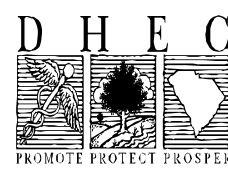

South Cardina Department of Health
and Eviromnental Control

\section{Cross Reference Report for QA and Analytes}

Analytical Method Reference:

Lab Reference (to Facility Sample):

Subject / Project:

Facility:
SW846 9056 - Inorganic Anions (TCLP Extraction BWXS - NELS (SDG 0310002)

Low-Curie Salt

Westinghouse Savannah River Compan

\begin{tabular}{|c|c|c|c|c|c|c|}
\hline LAB ID \# & FACILITY SAMP ID \# & TC EXTR BATCH \# & PREP BATCH \# & ANALYSIS BATCH \# & OTHER & COMMENTS \\
\hline S683-05B1 (PBLK64 - TCLP BLANK) & None & $683-05$ & Not Applicable & $110703 / 9056$ & --- & --- \\
\hline S683-05B1QC (PBLKQC64) & None & $683-05$ & Not Applicable & $110703 / 9056$ & --- & --- \\
\hline 0310002-11AMS & 41-TM5 & $683-05$ & Not Applicable & $110703 / 9056$ & --- & --- \\
\hline $0310002-08 \mathrm{~A}$ & 41-TM2 & $683-05$ & Not Applicable & 110703 / 9056 & --- & --- \\
\hline 0310002-09A & 41-TM3 & $683-05$ & Not Applicable & $110703 / 9056$ & --- & --- \\
\hline $0310002-10 \mathrm{~A}$ & 41-TM4 & $683-05$ & Not Applicable & $110703 / 9056$ & --- & --- \\
\hline 0310002-11ADUP & 41-TM5 & $683-05$ & Not Applicable & $110703 / 9056$ & --- & --- \\
\hline & & & & & & \\
\hline & & & & & & \\
\hline & & & & & & \\
\hline & & & & & & \\
\hline & & & & & & \\
\hline & & & & & & \\
\hline & & & & & & \\
\hline & & & & & & \\
\hline & & & & & & \\
\hline & & & & & & \\
\hline & & & & & & \\
\hline & & & & & & \\
\hline & & & & & & \\
\hline & & & & & & \\
\hline & & & & & & \\
\hline & & & & & & \\
\hline & & & & & & \\
\hline & & & & & & \\
\hline & & & & & & \\
\hline & & & & & & \\
\hline & & & & & & \\
\hline
\end{tabular}


WSRC-TR-2004-00051

Revision 0

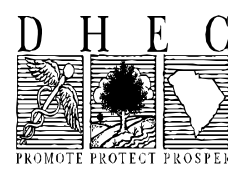

Souch Carolina Department of Health

\section{Cross Reference Report for QA and Analytes}

Analytical Method Reference:

Lab Reference (to Facility Sample):

Subject / Project:

Facility:
SW846 9056 - Inorganic Anions (Water Leach

BWXS - NELS (SDG 0310002)

Low-Curie Salt

Westinghouse Savannah River Compan

\begin{tabular}{|c|c|c|c|c|c|c|}
\hline LAB ID \# & FACILITY SAMP ID \# & TC EXTR BATCH \# & PREP BATCH \# & ANALYSIS BATCH \# & OTHER & COMMENTS \\
\hline S3215-66B1 (PBLK62 - LEACH BLANK) & None & Not Applicable & S3215-66 & 102603 / 9056 & --- & --- \\
\hline S3215-66S1 (LCS62) & None & Not Applicable & S3215-66 & $102603 / 9056$ & --- & --- \\
\hline 0310002-15AMS & 41-WA4 & Not Applicable & S3215-66 & $102603 / 9056$ & --- & --- \\
\hline 0310002-16AMSD & 41-WA5 & Not Applicable & S3215-66 & $102603 / 9056$ & --- & --- \\
\hline $0310002-12 \mathrm{~A}$ & 41-WA1 & Not Applicable & S3215-66 & 102603 / 9056 & --- & --- \\
\hline $0310002-13 \mathrm{~A}$ (DUP) & 41-WA2 & Not Applicable & S3215-66 & 102603 / 9056 & --- & --- \\
\hline $0310002-14 \mathrm{~A}$ & 41-WA3 & Not Applicable & S3215-66 & $102603 / 9056$ & --- & --- \\
\hline & & & & & & \\
\hline & & & & & & \\
\hline & & & & & & \\
\hline & & & & & & \\
\hline & & & & & & \\
\hline & & & & & & \\
\hline & & & & & & \\
\hline & & & & & & \\
\hline & & & & & & \\
\hline & & & & & & \\
\hline & & & & & & \\
\hline & & & & & & \\
\hline & & & & & & \\
\hline & & & & & & \\
\hline & & & & & & \\
\hline & & & & & & \\
\hline & & & & & & \\
\hline & & & & & & \\
\hline & & & & & & \\
\hline & & & & & & \\
\hline & & & & & & \\
\hline & & & & & & \\
\hline & & & & & & \\
\hline
\end{tabular}


WSRC-TR-2004-00051

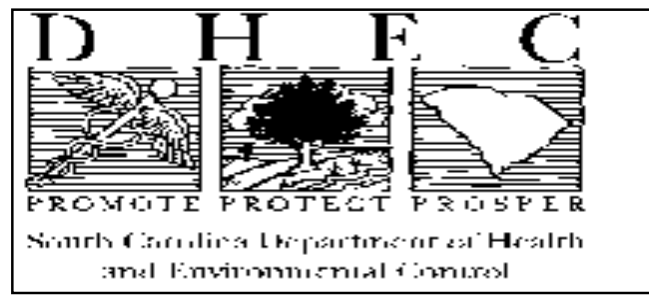

Type Data:

Company Name:

Subject/Project:

\section{Industrial RCRA - TCLP Semi-Volatiles}

Westinghouse Savannah River Company Low-Curie Salt

Facility Sample ID \#

Laboratory Sample ID \#

Laboratory Name

SC Laboratory Certification \#

Subcontracted Laboratory Certification \#

Subcontracted Laboratory Name

Laboratory Receipt Information (Chain of Custody Must be Attached)

\begin{tabular}{|c|c|c|c|c|c|c|c|c|c|c|c|c|c|}
\hline \multicolumn{9}{|c|}{ Laboratory Receipt Information (Chain of Custody Must be Attached) } & Attacced & Attached & Attached & & 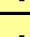 \\
\hline \multicolumn{9}{|c|}{ Semi-Volatile Organic Compounds } & Subcontract ${ }^{1}$ & & & Subcontract ${ }^{1}$ & \\
\hline Analytical Analaytes & $\begin{array}{l}\text { Preparation } \\
\text { Method }\end{array}$ & \begin{tabular}{|c|} 
Analytical \\
Method
\end{tabular} & $\begin{array}{l}\text { Detection } \\
\text { Limit }(\mathrm{mg} / \mathrm{l})\end{array}$ & $\begin{array}{l}\text { Quantitation } \\
\text { Limit (ma/l) }\end{array}$ & MCL $(\mathrm{mg} / \mathrm{l})$ & $\begin{array}{c}10 \times \mathrm{MCL} \\
(\mathrm{mg} / \mathrm{l})\end{array}$ & $\begin{array}{r}30 \times \mathrm{MCL} \\
(\mathrm{mg} / \mathrm{ll})\end{array}$ & - & \multicolumn{5}{|c|}{ - } \\
\hline 1,4-Dichlorobenzene & 3550 & SW8270C & - & - & - & - & - & - & - & - & - & - & \\
\hline -Cresol (2-Methtiphenol) & 3550 & SW8270C & - & - & - & - & - & - & - & - & - & - & \\
\hline n-and p-Cresol (3-\& 4-Methylphenol) & 3550 & SW8270C & - & - & - & - & - & - & - & - & - & - & - \\
\hline Texachloroethane & 3550 & SW8270C & - & - & - & - & - & - & - & - & - & - & \\
\hline Vitrobenzene & 3550 & SW8270C & - & - & - & - & - & - & - & - & - & - & - \\
\hline Hexochloro-1,3-butadiene & 3550 & SW8270C & - & - & - & - & - & - & - & - & - & - & - \\
\hline $2,4,6$-Trichlorophenol & 3550 & SW8270C & - & - & - & - & - & - & - & - & - & - & 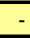 \\
\hline 2,4,5-Trichlorophenol, etc. & 3550 & SW8270C & - & - & - & - & - & - & - & - & - & - & - \\
\hline 2,4-Dinitrotoluene & 3550 & SW8270C & - & - & - & - & - & - & - & - & - & - & - \\
\hline exachlorobenzene & 3550 & SW8270C & - & - & - & - & - & - & - & - & - & & \\
\hline entachlorophenol & 3550 & SW8270C & - & - & - & - & - & - & - & - & - & - & 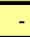 \\
\hline Dyridine & 3550 & SW8270C & - & - & - & - & - & - & - & - & - & - & - \\
\hline henol ${ }^{2}$ & 3550 & SW8270C & 0.05 & 1.0 & 22000 & 220000 & $6.6 \mathrm{E}+05$ & - & $<1.0$ & $<1.0$ & $<1.0$ & - & - \\
\hline \multicolumn{9}{|c|}{ Quality Assurance (for above samples) } & \\
\hline \multirow{2}{*}{\multicolumn{9}{|c|}{$\begin{array}{l}\text { TCLP Extraction Batch \# } \\
\text { Semivolatile Extraction Batch \# }\end{array}$}} & $683-05$ & $683-05$ & $683-05$ & - & \\
\hline & & & & & & & & & $648-66$ & $648-66$ & $648-66$ & - & \\
\hline \multicolumn{9}{|l|}{ Analysis Batch Number } & 102603.B & 102603.B & $102603 . \mathrm{B}$ & - & \\
\hline \multicolumn{9}{|l|}{ Surrogates. \% Recovery } & $\mathrm{A}$ & $\mathrm{A}$ & $\mathrm{A}$ & - & - \\
\hline \multirow{2}{*}{\multicolumn{9}{|c|}{$\frac{\text { Nitrobenzene, d5 }}{\text { 2-Fluorobiphenol }}$}} & & & & & \\
\hline & & & & & & & & & - & - & - & - & \\
\hline \multicolumn{9}{|l|}{ Terphenyl, d14 } & & - & - & - & - \\
\hline \multirow{2}{*}{\multicolumn{9}{|c|}{$\frac{\text { Phenol, d6 }}{\text { 2-Fluorophenol }}$}} & 34 & 31 & 34 & - & \\
\hline & & & & & & & & & & & & & \\
\hline \multicolumn{9}{|l|}{ 2, $2,4,6$-Tribromophenol } & & & & & \\
\hline
\end{tabular}

2. PRG is given instead of the MCL. No MCL has been specified for this analyte.

Results in Milligrams per Liter Waste Stream 1

\begin{tabular}{|c|c|c|}
\hline 41-TM1 & 41-TM2 & 41-TM3 \\
\hline 0310002-07A & 0310002-08A & 0310002-09A \\
\hline
\end{tabular}

310002-09A

BWXS-NELS BWXS-NELS BWXS-NELS

Pending

BWXS-NELS

\section{Revision 0}

Date: $\underline{01 / 30 / 04}$

(1)


WSRC-TR-2004-00051

Revision 0

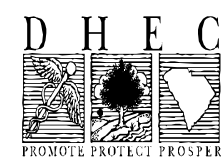

CT PROSPE

South Gardina Departurnut of Healh
and Invironmental Control
METHOD NAME:

SUBJECT:

Industrial RCRA - TCLP Semi-Volatiles BWXT 1-1999-0039
Reference:

Instrument:

Analyte Concentrations, $\mathrm{mg} / \mathrm{I}$

\begin{tabular}{|c|c|c|c|c|c|c|c|c|c|c|c|c|c|c|c|c|c|}
\hline & \multicolumn{8}{|c|}{ Analyte Concentrations, mg / I } & \multicolumn{8}{|c|}{ Recovery Percent } & Flags \\
\hline Analytes & RDL & MDL & Blank & LCS & LCSD & MS & MSD & Other & LCS & LCSD & MS & MSD & Ave MS/MSD & REC Limits & $\%$ RPD & RPD Limits & - \\
\hline 1,4-Dichlorobenzene & - & - & - & - & - & - & - & - & - & - & - & - & - & - & - & - & - \\
\hline o-Cresol (2-Methtlphenol) & - & - & - & - & - & - & - & - & - & - & - & - & - & - & - & - & - \\
\hline $\mathrm{m}$ - and p-Cresol (3- \& 4-Methylphenol) & - & - & - & - & - & - & - & - & - & - & - & - & - & - & - & - & - \\
\hline Hexachloroethane & - & - & - & - & - & - & - & - & - & - & - & - & - & - & - & - & - \\
\hline Nitrobenzene & - & - & - & - & - & - & - & - & - & - & - & - & - & - & - & - & - \\
\hline Hexochloro-1,3-butadiene & - & - & - & - & - & - & - & - & - & - & - & - & - & - & - & - & - \\
\hline 2,4,6-Trichlorophenol & - & - & - & $=$ & - & - & - & 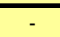 & - & 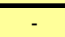 & 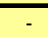 & - & - & - & - & - & - \\
\hline 2,4,5-Trichlorophenol, etc. & - & 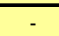 & 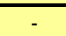 & - & - & 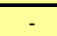 & 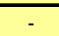 & 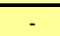 & 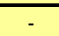 & 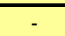 & - & 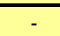 & - & 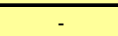 & - & - & - \\
\hline 2,4-Dinitrotoluene & - & - & 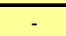 & - & - & 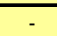 & 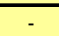 & 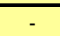 & 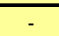 & 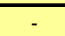 & - & 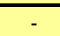 & 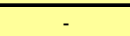 & $\overline{-1}$ & - & - & - \\
\hline Hexachlorobenzene & - & - & - & - & - & - & - & - & - & - & - & - & - & - & - & - & - \\
\hline Pentachlorophenol & - & - & - & - & - & - & - & - & - & - & - & - & - & - & - & - & - \\
\hline Pyridine & - & - & - & - & - & - & - & - & - & - & - & - & - & - & - & - & 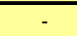 \\
\hline Phenol2 & 22000 & 0.05 & $<1.0$ & 410 & - & 7.50 & 7.50 & - & 95 & - & 42 & 36 & 39 & $12-110$ & 15 & $42 \%$ & - \\
\hline
\end{tabular}


WSRC-TR-2004-00051

Revision 0

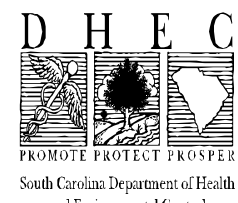

South Carolina Departrent of Heal

\section{Cross Reference Report for QA and Analytes}

Analytical Method Reference:

Lab Reference (to Facility Sample):

Subject / Project:

Facility:
SW846 3510C / 8270C

BWXS - NELS (SDG 0310002

Low-Curie Salt

Westinghouse Savannah River Company

\begin{tabular}{|c|c|c|c|c|c|c|}
\hline LAB ID \# & FACILITY SAMP ID \# & TC EXTR BATCH \# & NV EXTR BATCH \# & ANALYSIS BATCH \# & OTHER & COMMENTS \\
\hline S648-66B1 (SBLK31 - EXT BLANK) & None & $683-05$ & $648-66$ & $102603 . \mathrm{B}$ & --- & --- \\
\hline T648-66B2 (SBLK32 - METHOD BLANK) & None & $683-05$ & 648-66 & 102603.B & $\begin{array}{c}-- \\
-\end{array}$ & $\begin{array}{c}-- \\
-\end{array}$ \\
\hline W648-66S1 (LCS31) & None & $683-05$ & $648-66$ & 102603.B & --- & --- \\
\hline 0310002-10AMS & 41-TM4 & $683-05$ & $648-66$ & 102603.B & --- & --- \\
\hline 0310002-11AMSD & 41-TM5 & $683-05$ & $648-66$ & 102603.B & 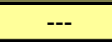 & 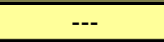 \\
\hline $0310002-07 \mathrm{~A}$ & 41-TM1 & $683-05$ & $648-66$ & 102603.B & --- & --- \\
\hline $0310002-08 \mathrm{~A}(\mathrm{DUP})$ & 41-TM2 & $683-05$ & $648-66$ & 102603.B & --- & --- \\
\hline 0310002-09A & 41-TM3 & $683-05$ & $648-66$ & 102603.B & --- & --- \\
\hline & & & & & & \\
\hline & & & & & & \\
\hline & & & & & & \\
\hline & & & & & & \\
\hline & & & & & & \\
\hline & & & & & & \\
\hline & & & & & & \\
\hline & & & & & & \\
\hline & & & & & & \\
\hline & & & & & & \\
\hline & & & & & & \\
\hline & & & & & & \\
\hline & & & & & & \\
\hline & & & & & & \\
\hline & & & & & & \\
\hline & & & & & & \\
\hline & & & & & & \\
\hline & & & & & & \\
\hline & & & & & & \\
\hline & & & & & & \\
\hline & & & & & & \\
\hline
\end{tabular}


WSRC-TR-2004-00051

Revision 0

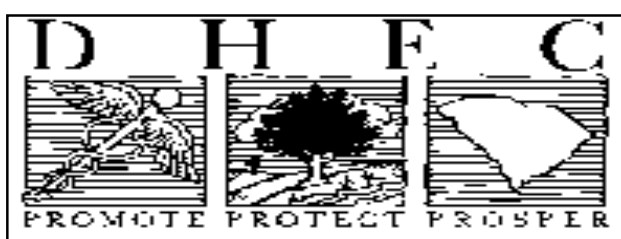

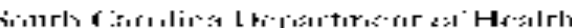

anel linvirimentalianises
Type Data: Industrial RCRA - TCLP Volatiles

Company Name: Westinghouse Savannah River Company

Subject/Project:
Low-Curie Salt

Facility Sample ID \#

Laboratory Sample ID \#

Laboratory Name

SC Laboratory Certification \#

Subcontracted Laboratory Certification \#

Subcontracted Laboratory Name

Laboratory Receipt Information (Chain of Custody Must be Attached)

\begin{tabular}{|c|c|c|c|c|c|c|c|c|c|c|c|c|c|}
\hline \multicolumn{4}{|c|}{ 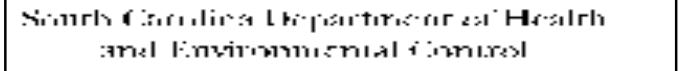 } & & & & & & \multicolumn{5}{|c|}{ Results in Milligrams per Liter } \\
\hline & & & & & & & & & \multicolumn{3}{|c|}{ Waste Stream 1} & \multirow[b]{2}{*}{ - } & \multirow[b]{2}{*}{-} \\
\hline & & & & & & & & & 9/29/2003 & 9/29/2003 & 9/29/2003 & & \\
\hline \multicolumn{9}{|l|}{ Facility Sample ID \# } & 41-TV1 & 41-TV2 & 41-TV3 & - & - \\
\hline \multicolumn{9}{|c|}{ Laboratory Sample ID \# } & 0310002-02AT3 & 0310002-03AT3 & 0310002-04AT3 & - & - \\
\hline \multicolumn{9}{|c|}{ Laboratory Name } & BWXS-NELS & BWXS-NELS & BWXS-NELS & - & - \\
\hline \multicolumn{9}{|c|}{ SC Laboratory Certification \# } & Pending & Pending & Pending & - & - \\
\hline \multicolumn{9}{|c|}{ Subcontracted Laboratory Certification \# } & --- & --- & --- & - & - \\
\hline \multicolumn{9}{|c|}{ Subcontracted Laboratory Name } & $-\ldots$ & $-\ldots$ & 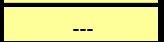 & - & - \\
\hline \multicolumn{9}{|c|}{ Laboratory Receipt Information(Chain of Custody Must be Attached) } & --- & $-\ldots$ & --- & - & - \\
\hline \multicolumn{9}{|c|}{ TCLP Volatile Organic Compounds } & Subcontract ${ }^{1}$ & - & - & Subcontract ${ }^{1}$ & - \\
\hline Analytical Parameter & $\begin{array}{c}\begin{array}{c}\text { Preparation } \\
\text { Method }\end{array} \\
\end{array}$ & \begin{tabular}{|c|}
$\begin{array}{c}\text { Analytical } \\
\text { Method }\end{array}$ \\
\end{tabular} & \begin{tabular}{c|} 
Detection \\
Limit (mg/l) \\
\end{tabular} & $\begin{array}{l}\text { Quantitation } \\
\text { Limit (mg/l) }\end{array}$ & $\begin{array}{l}\mathrm{MCL} \\
(\mathrm{mg} / \mathrm{ll})\end{array}$ & $\begin{array}{c}10 \times \mathrm{MCL} \\
(\mathrm{mg} / \mathrm{ll})\end{array}$ & \begin{tabular}{|c|}
$\begin{array}{c}30 \times \mathrm{MCL} \\
(\mathrm{mg} / \mathrm{ll})\end{array}$ \\
\end{tabular} & - & \\
\hline Benzene & $5030 \mathrm{~B}$ & SW8260B & 0.001 & 0.01 & 0.005 & 0.05 & \begin{tabular}{|l|l|}
0.15 \\
\end{tabular} & - & $<0.010$ & $2.9 \mathrm{E}-3(\mathrm{~J})$ & $<0.010$ & - & - \\
\hline Carbon Tetrachloride & $5030 \mathrm{~B}$ & SW8260B & - & - & - & - & - & - & - & - & - & - & - \\
\hline Tetrachloroethylene & $5030 \mathrm{~B}$ & SW8260B & - & - & - & - & - & - & - & - & - & - & - \\
\hline Trichloroethylene & $5030 \mathrm{~B}$ & SW8260B & - & - & - & - & - & - & - & - & - & - & - \\
\hline Vinyl Chloride & $5030 \mathrm{~B}$ & SW8260B & - & - & - & - & - & - & - & - & - & - & - \\
\hline 1.1-Dichloroethylene & $5030 \mathrm{~B}$ & SW8260B & - & - & - & - & - & - & - & - & - & - & - \\
\hline 1,2-Dichloroethane & $5030 \mathrm{~B}$ & SW8260B & - & - & - & - & - & - & - & - & - & - & - \\
\hline Chloroform & $5030 \mathrm{~B}$ & SW8260B & - & - & - & - & - & - & - & - & - & - & - \\
\hline MEK (2-Butanone) & $5030 \mathrm{~B}$ & SW8260B & - & - & - & - & - & - & - & - & - & - & - \\
\hline Chlorobenzene & $5030 \mathrm{~B}$ & SW8260B & - & - & - & - & - & - & - & - & - & - & - \\
\hline Toluene & $5030 \mathrm{~B}$ & SW8260B & 0.002 & 0.01 & 1 & 10 & 30 & - & $1.2 \mathrm{E}-3(\mathrm{~J})$ & $<0.010$ & $<0.010$ & - & - \\
\hline 1-Butanol ${ }^{2}$ & $5030 \mathrm{~B}$ & SW8260B & 0.100 & 0.1 & 3600 & 36000 & $1.1 \mathrm{E}+05$ & - & $<0.1$ & $<0.1$ & $<0.1$ & - & \\
\hline \multicolumn{14}{|c|}{ Quality Assurance (for above samples) } \\
\hline \multicolumn{9}{|c|}{ TCLP ZHE Extraction Batch \# } & $683-05$ & $683-05$ & $683-05$ & 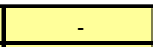 & - \\
\hline \multicolumn{9}{|c|}{ Volatile Analysis Batch \# } & $100803 . \mathrm{B}$ & $100803 . \mathrm{B}$ & 100803.B & - & - \\
\hline \multicolumn{9}{|c|}{ Surrogates, \% Recovery } & $\mathrm{A}$ & $\mathrm{NA}$ (toluene) & $\mathrm{A}$ & - & - \\
\hline \multicolumn{9}{|c|}{ 1,2- Dichlorethane, d4 } & - & - & - & - & - \\
\hline \multicolumn{9}{|c|}{ Toluene, d8 } & 104 & 68 & 103 & - & - \\
\hline \multicolumn{9}{|c|}{ 4-Bromofluorobenzene } & 96 & 106 & 98 & - & - \\
\hline \multicolumn{9}{|c|}{ Other } & 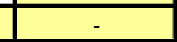 & - & & - & - \\
\hline
\end{tabular}

1. Subcontracted Laboratory Used for these Parameters(Analytes)

2. PRG is given instead of the MCL. No MCL has been specified for this analyte.

(J) indicates values that were above the method detection limits but below the quantitation limits. 
WSRC-TR-2004-00051

Revision 0

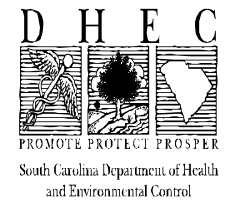

Reference:

Instrument:
LABORATORY:
METHOD NAME:
SUBJECT:

BWXS - NELS

SW-846-5030B and SW-846-8260B

QA- Blk, Laboratory Control Sample (LCS), Matrix Spike (MS), Matrix Spike Duplicate (MSD)

Date: $\quad 01 / 30 / 04$

\begin{tabular}{|c|c|c|c|c|c|c|c|c|c|c|c|c|c|c|c|c|c|}
\hline & \multicolumn{8}{|c|}{ Analyte Concentrations, $\mathrm{mg} / \mathrm{I}$} & \multicolumn{8}{|c|}{ Recovery Percent } & \multirow{2}{*}{$\begin{array}{c}\text { Flag } \\
-\end{array}$} \\
\hline Analytes & RDL & MDL & Blank & LCS & LCSD & MS & MSD & Other & LCS & LCSD & MS & MSD & Ave MS/MSD & \begin{tabular}{|l|} 
REC Limits \\
\end{tabular} & $\%$ RPD & RPD Limits & \\
\hline Benzene & 0.005 & 0.001 & $2.2 \mathrm{e}-3(\mathrm{~J})$ & 0.041 & - & 0.041 & 0.041 & - & 84 & - & 96 & 95 & 95.5 & $37-151$ & 1 & $21 \%$ & - \\
\hline Carbon Tetrachloride & - & - & - & - & - & - & - & - & - & - & - & - & - & - & - & - & - \\
\hline Tetrachloroethylene & - & - & - & - & - & - & - & - & - & - & - & - & - & - & - & - & - \\
\hline Trichloroethylene & - & - & - & - & - & - & - & - & - & - & - & - & - & - & - & - & - \\
\hline Vinyl Chloride & - & - & - & - & - & - & - & - & - & - & - & - & - & - & - & - & - \\
\hline 1.1-Dichloroethylene & - & - & - & - & - & - & - & - & - & - & - & - & - & - & - & - & - \\
\hline 1,2-Dichloroethane & - & - & - & - & - & - & - & - & - & - & - & - & - & - & - & - & - \\
\hline Chloroform & - & - & - & - & - & - & - & - & - & - & - & - & - & - & - & - & - \\
\hline MEK (2-Butanone) & - & - & - & - & - & - & - & - & - & - & - & - & - & - & - & - & - \\
\hline Chlorobenzene & - & - & - & - & - & - & - & - & - & - & - & - & - & - & - & - & - \\
\hline Toluene & 1 & 0.002 & $<0.010$ & 0.041 & - & 0.041 & 0.041 & - & 84 & - & 28 & 105 & 67 & $47-150$ & 116 & $21 \%$ & - \\
\hline 1-Butanol2 & 3600 & 0.100 & $<0.10$ & 0.41 & - & 0.41 & 0.41 & - & 95 & - & 87 & 96 & 92 & $47-150$ & 10 & $21 \%$ & - \\
\hline
\end{tabular}


WSRC-TR-2004-00051

Revision 0

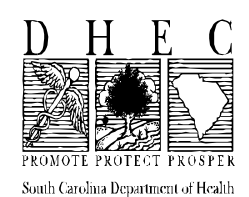

Sounh Carodina Departricent of Healib

\section{Cross Reference Report for QA and Analytes}

Analytical Method Reference:

Lab Reference (to Facility Sample):

Subject / Project:

Facility:

SW846 5030B / 8260B

BWXS - NELS (SDG 0310002)

Low-Curie Salt

Westinghouse Savannah River Company

LAB ID \#

\begin{tabular}{|c|c|c|c|}
\hline FACILITY SAMP ID \# & TC EXTR BATCH \# & DIGESTION BATCH \# & ANALYSIS BATCH \#
\end{tabular}

\begin{tabular}{|c|c|c|c|c|c|c|}
\hline LAB ID \# & FACILITY SAMP ID \# & TC EXTR BATCH \# & DIGESTION BATCH \# & ANALYSIS BATCH \# & OTHER & COMMENTS \\
\hline S683-05B2 (VBK53 - ZHE EXT BLANK) & None & $683-05$ & Not Applicable & $100803 . \mathrm{B}$ & --- & --- \\
\hline FE01 (VBLK51 - METHOD BLANK) & None & $683-05$ & Not Applicable & 100803.B & --- & --- \\
\hline FFD01 (VBLK52 - METHOD BLANK) & None & $683-05$ & Not Applicable & $100803 . \mathrm{B}$ & --- & --- \\
\hline FG01 (VBLK54 - METHOD BLANK) & None & $683-05$ & Not Applicable & 100803.B & --- & --- \\
\hline S4100 (LCS51) & None & $683-05$ & Not Applicable & 100803.B & --- & --- \\
\hline FF01LCS (LCS52) & None & $683-05$ & Not Applicable & 100803.B & --- & --- \\
\hline FG01LCS (LCS54) & None & $683-05$ & Not Applicable & 100803.B & --- & --- \\
\hline 0310002-05AT3MS & 41-TV4 & $683-05$ & Not Applicable & $100803 . B$ & --- & --- \\
\hline 0310002-06AT3MSD & $41-T V 5$ & $683-05$ & Not Applicable & 100803.B & --- & --- \\
\hline 0310002-02AT3 & 41-TV1 & $683-05$ & Not Applicable & $100803 . B$ & --- & --- \\
\hline 0310002-03AT3 (DUP) & 41-TV2 & $683-05$ & Not Applicable & 100803.B & --- & --- \\
\hline 0310002-04AT3 & $41-T V 3$ & $683-05$ & Not Applicable & 100803.B & --- & --- \\
\hline & & & & & & \\
\hline & & & & & & \\
\hline & & & & & & \\
\hline & & & & & & \\
\hline & & & & & & \\
\hline & & & & & & \\
\hline & & & & & & \\
\hline & & & & & & \\
\hline & & & & & & \\
\hline & & & & & & \\
\hline & & & & & & \\
\hline & & & & & & \\
\hline & & & & & & \\
\hline & & & & & & \\
\hline & & & & & & \\
\hline & & & & & & \\
\hline & & & & & & \\
\hline & & & & & & \\
\hline
\end{tabular}


Data Report For: Westinghouse SRTC-ADS

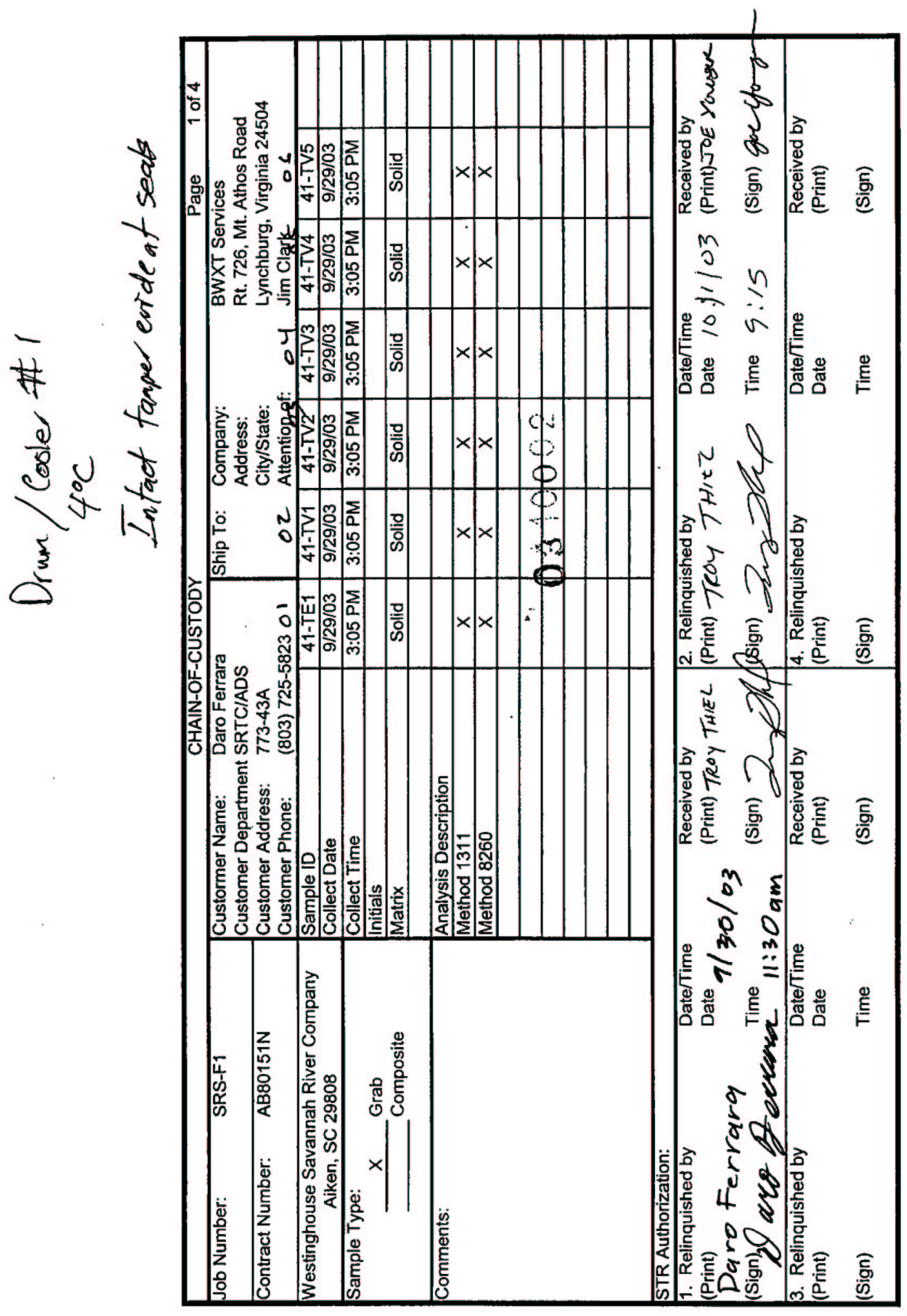

BWXT Services, Inc. - NEL Services • 2016 Mt. Athos Rd. • Lynchburg, VA • 24504-5447 • (434) 522-5165 
Data Report For: Westinghouse SRTC-ADS

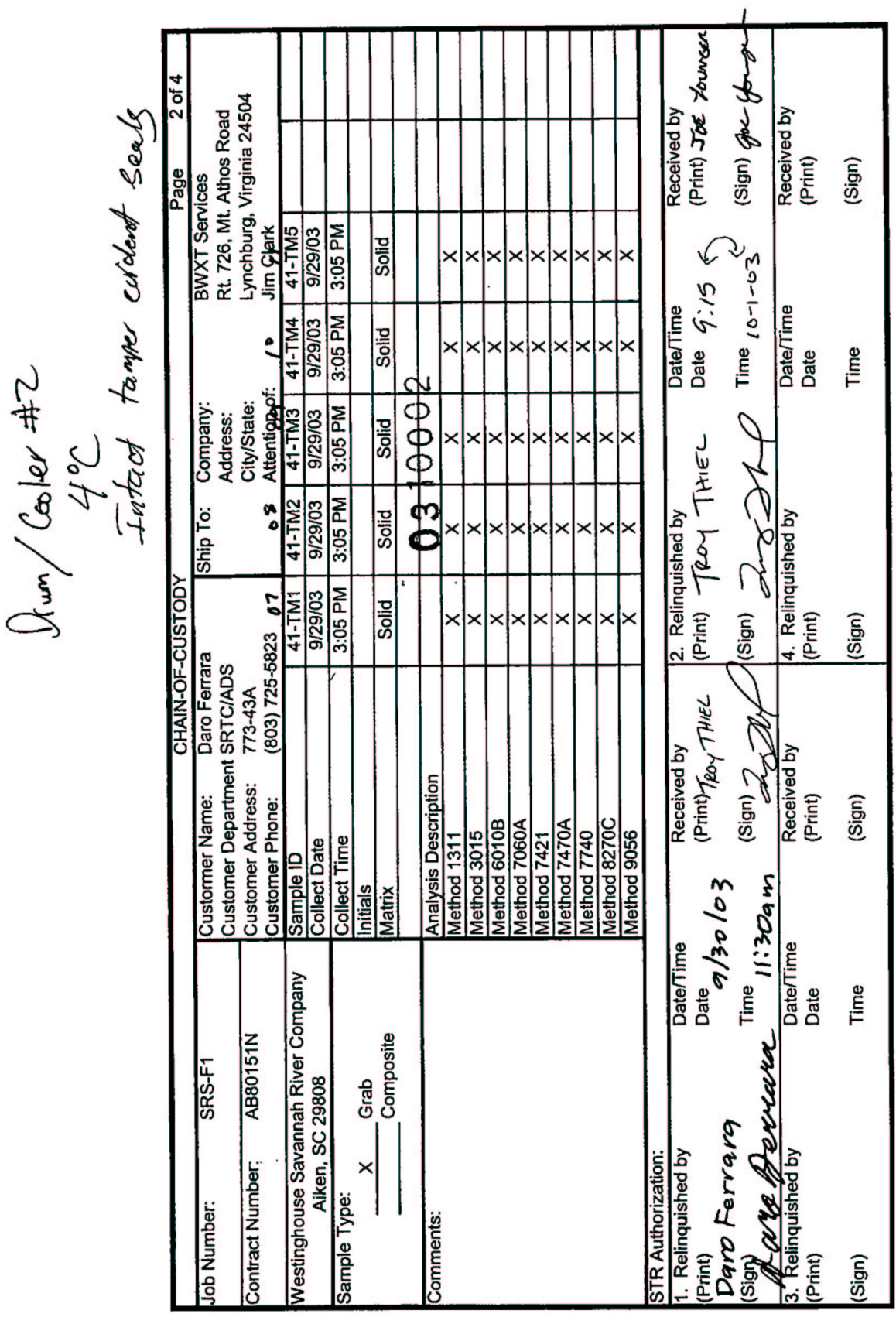

BWXT Services, Inc. - NEL Services • 2016 Mt. Athos Rd. • Lynchburg, VA • 24504-5447 • (434) 522-5165 
Data Report For: Westinghouse SRTC-ADS

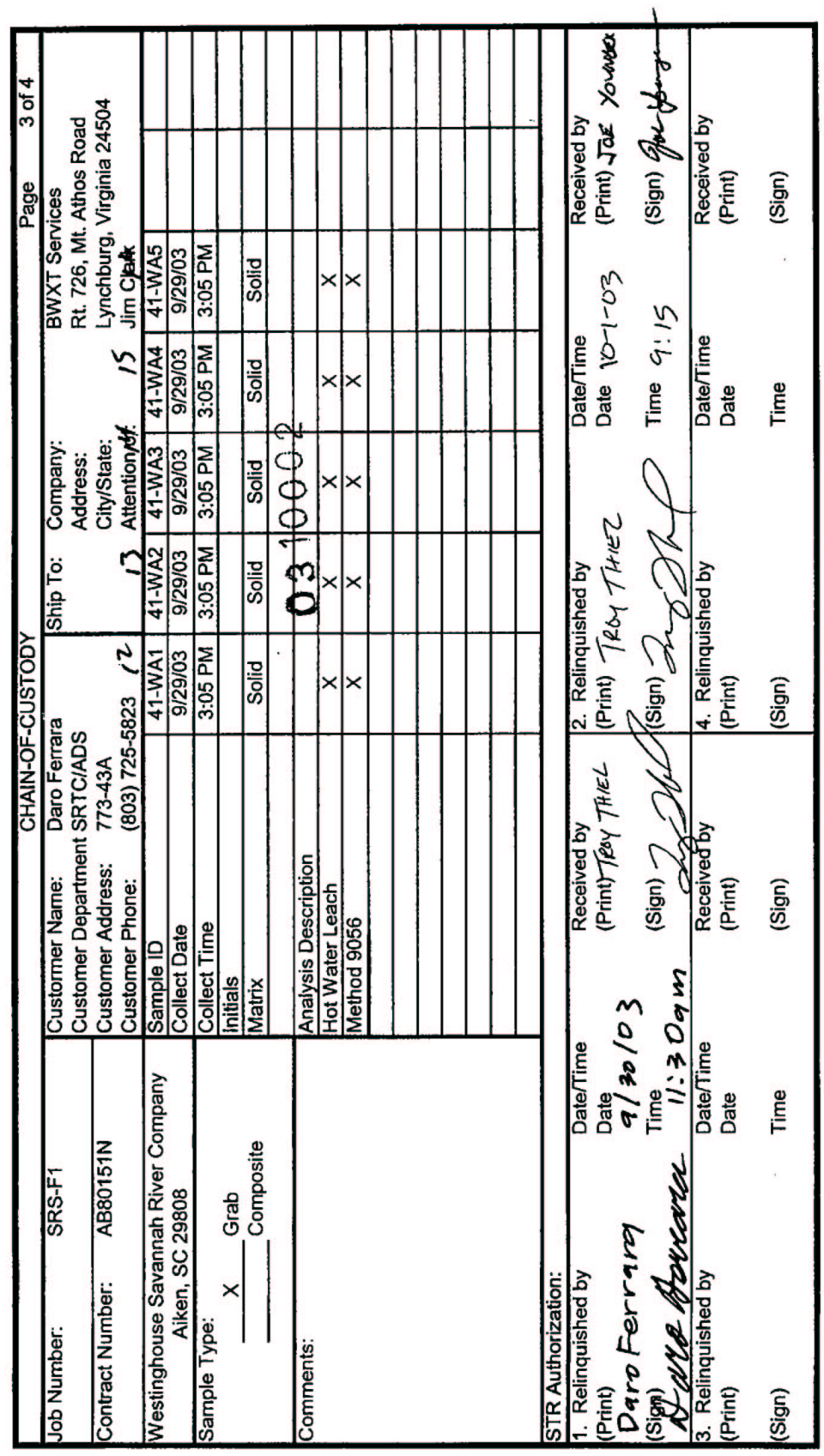

BWXT Services, Inc. - NEL Services • 2016 Mt. Athos Rd. • Lynchburg, VA • 24504-5447 • (434) 522-5165 
Data Report For: Westinghouse SRTC-ADS

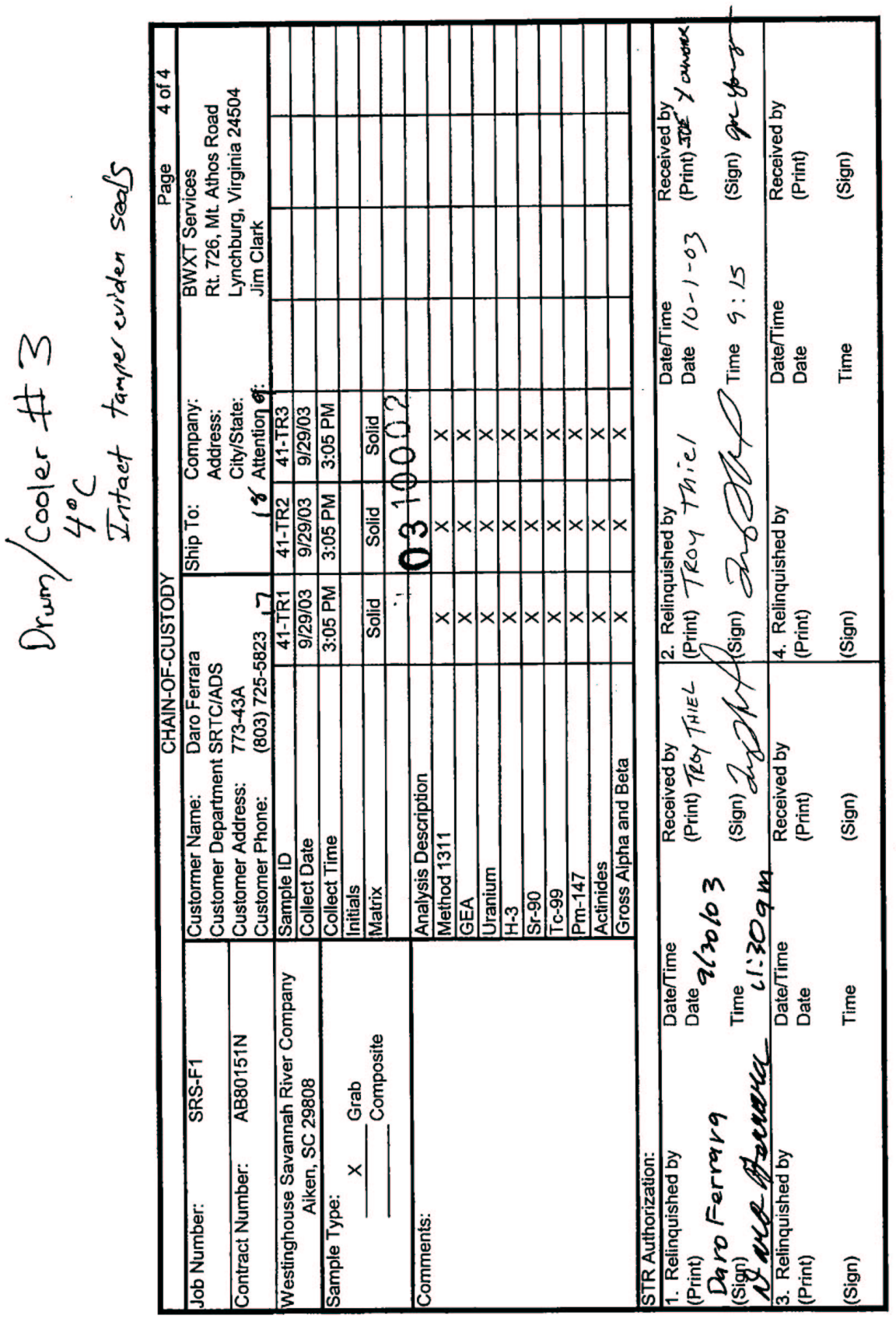

BWXT Services, Inc. - NEL Services • 2016 Mt. Athos Rd. • Lynchburg, VA • 24504-5447 • (434) 522-5165 